\title{
Identification of key genes for guiding chemotherapeutic management in ovarian cancer using translational bioinformatics
}

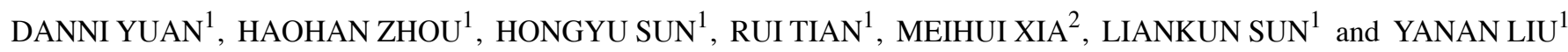 \\ ${ }^{1}$ Key Laboratory of Pathobiology, Department of Pathophysiology, Ministry of Education, College of Basic Medical Sciences; \\ ${ }^{2}$ Department of Obstetrics, First Hospital, Jilin University, Changchun, Jilin 130021, P.R. China
}

Received November 2, 2019; Accepted April 1, 2020

DOI: $10.3892 / \mathrm{ol} .2020 .11672$

\begin{abstract}
The emergence of resistance to chemotherapy drugs in patients with ovarian cancer is still the main cause of low survival rates. The present study aimed to identify key genes that may provide treatment guidance to reduce the incidence of drug resistance in patients with ovarian cancer. Original data of chemotherapy sensitivity and chemoresistance of ovarian cancer were obtained from the Gene Expression Omnibus dataset GSE73935. Differentially expressed genes (DEGs) between sensitive and resistant ovarian cancer cell lines were screened by Empirical Bayes methods. Overlapping DEGs between four chemoresistant groups were identified by Venn map analysis. Protein-protein interaction networks were also constructed, and hub genes were identified. The hub genes were verified by in vitro experiments as well as The Cancer Genome Atlas data. Results from the present study identified eight important genes that may guide treatment decisions regarding chemotherapy regimens for ovarian cancer, including epidermal growth factor-like repeats and discoidin I-like domains 3, NRAS proto-oncogene, hyaluronan and proteoglycan link protein 1 , activated protein $\mathrm{C}$ receptor, CD53, cyclin-dependent kinase inhibitor $2 \mathrm{~A}$, insulin-like growth factor 1 receptor and roundabout guidance receptor 2 genes. Their expressions were found to have an impact on the prognosis of different treatment groups (cisplatin, paclitaxel, cisplatin + paclitaxel, cisplatin + doxorubicin and cisplatin + topotecan). The results indicated that these genes may minimise the occurrence of ovarian cancer drug resistance and may provide effective treatment options for patients with ovarian cancer.
\end{abstract}

Correspondence to: Professor Liankun Sun or Professor Yanan Liu, Key Laboratory of Pathobiology, Department of Pathophysiology, Ministry of Education, College of Basic Medical Sciences, Jilin University, 126 Xinmin Street, Changchun, Jilin 130021, P.R. China E-mail:sunlk@jlu.edu.cn

E-mail: ynliu@jlu.edu.cn

Key words: ovarian cancer, chemotherapy resistance, bioinformatics, precision medicine

\section{Introduction}

Ovarian cancer is one of the most serious threats to reproductive health in women, which is estimated to account for 21,750 new cases and 13,940 mortalities in the United States in 2020, with the highest mortality rate among female reproductive system malignancies (1). For patients initially diagnosed with ovarian epithelial cancer, the standard treatment is tumour resection and chemotherapy (2). Carboplatin combined with paclitaxel is currently the standard first-line chemotherapy for patients with ovarian cancer (3), whereas carboplatin combined with pegylated liposomal doxorubicin is considered as an alternative to standard therapy (4). Alternatively, carboplatin plus topotecan co-treatment is recommended in patients who are allergic to paclitaxel (5). However, owing to the differences observed between individual patients with ovarian cancer and due to tumour heterogeneity, patient sensitivity and tolerance to different drugs differ (6). Thus, improved treatment plans for precision medicine would benefit patient outcome. Considering the different mechanisms of action of topotecan and three other common drugs, including cisplatin, doxorubicin and paclitaxel (7-10), the National Cancer Institute Clinical Trial Group in collaboration with the European Cancer Research and Treatment Organization successfully completed a phase II clinical trial to evaluate the triple combination of cisplatin, topotecan and paclitaxel in patients with advanced epithelial ovarian cancer (11). However, Brotto et al (12) and Hoskins et al (13) found that the combination of these three drugs did not significantly improve the quality of life of patients compared with the standard drug regimen of carboplatin plus paclitaxel, and increased the side effects experienced by the patients. Therefore, considering the efficacy and side effects, identifying the optimal combination of two drugs for individual patients is very important.

Clinical trials are the best method of studying the efficacy of chemotherapy regimens (14), but these are time-consuming and precision medicine is difficult to achieve (15). A phase III clinical trial conducted in Italy found that the overall survival and efficacy of carboplatin combined with doxorubicin is not superior to carboplatin plus paclitaxel; besides, different drug combinations display different side effects (16). It is recommended that drugs are selected based on patient tolerance to side effects. Another phase III clinical trial in France found that the progression-free survival (PFS) and efficacy of carboplatin 
combined with doxorubicin was superior to carboplatin plus paclitaxel in elderly patients (17). A number of phase III clinical trials found that carboplatin combined with topotecan reduced side effects in patients, whereas progression-free survival and overall survival were not higher compared with carboplatin plus paclitaxel $(18,19)$. However, this large-scale clinical trial was not suitable for individualised treatment due to tumour heterogeneity. Therefore, the data analysis methods presented in the current study may provide ideas and directions for basic research as well as indicate a design for future clinical trials.

To date, many data analysis studies on the expression profiles of common chemotherapeutic drug resistance genes in ovarian cancer have been performed. As reviewed by Galluzzi et al (20), these studies aimed to screen crucial molecules to reverse the drug resistance of ovarian cancer using corresponding target inhibitors or activators to restore chemosensitivity of tumour cells. Currently, the relationship between differentially expressed genes (DEGs) and clinical medication guidance has not yet been determined. Through the integrated analysis of gene expression profiling microarray data of four first-line chemotherapy drugs, cisplatin, doxorubicin, paclitaxel and topotecan, the present study aimed to identify effective and reliable molecular markers to provide guidance for the selection of clinical chemotherapy drugs. In this study, overlapping DEGs between the four different groups of drug-resistant ovarian cancer cells were identified by Venn map analysis, protein-protein interaction networks were generate, associated hub genes were identified and the accuracy of these hub genes was verified by in vitro experiments and data from The Cancer Genome Atlas (TCGA), which were used to determine possible effective treatment options for clinical treatment of patients with ovarian cancer.

\section{Materials and methods}

Microarray data. The GSE73935 microarray dataset was downloaded from the Gene Expression Omnibus (GEO) database (http://www.ncbi.nlm.nih.gov/geo). These RNA profiles were based on GPL13667 (HG-U219) Affymetrix human genome U219 beadchip platform containing a total of six cisplatin-resistant ovarian cell sublines (A2780), six doxorubicin-resistant cell sublines, six paclitaxel-resistant cell sublines, six topotecan-resistant cell sublines and three sensitive control cell sublines.

Identification of DEGs. Following data standardisation, the 'limma' package in $\mathrm{R}$ software (version 3.5, https://www.r-project.org) was applied to screen DEGs between the four groups of resistant ovarian cancer cell lines and the sensitive controls. Genes with $\mathrm{P}<0.01$ and $\log _{2}$ [fold change $(\mathrm{FC})] \mathrm{l}>2$ were considered as DEGs. Considering that the first line of clinical therapy is the combination of two drugs, the DEGs in each group were classified and further overlapped using the 'VennDiagram' package within R software. The genes that were upregulated or downregulated in several drug-resistant cell lines under the same classification were classified as overlapping DEGs. The combination of drugs mainly included cisplatin combined with doxorubicin, paclitaxel or topotecan, and paclitaxel combined with doxorubicin or topotecan.
Gene Ontology (GO) functional term and Kyoto Encyclopedia of Genes and Genomes (KEGG) pathway enrichment analysis. To determine the potential biological functions of the overlapping DEGs, GO (http://www.geneontology.org) term enrichment analysis was performed based on three aspects; biological process (BP), molecular function (MF) and cellular component (CC). Subsequently KEGG (http://www. genome.jp/kegg) pathway enrichment analysis was conducted to investigate the potential signalling pathways related to the overlapping DEGs. GO term and KEGG pathway enrichment analyses were performed using $\mathrm{R}$ software.

Protein-protein interaction (PPI) network construction and hub gene analysis. The potential interactions of the overlapping DEGs were analysed using STRING (https://string-db. org) software tool. PPI score was set to 0.4 , and all isolated nodes were hidden. Subsequently, the PPI network was visualised and further analysed using Cytoscape software (www. cytoscape.org). The 'CentiScaPe' plug-in of Cytoscape was used to calculate the degree and betweenness parameters of the PPI network; it was stipulated that a gene with degree $\geq$ mean $_{\text {degree }}+$ standard deviation $\left(\mathrm{SD}_{\text {degree }}\right.$ ) satisfying betweenness $\geq$ mean $_{\text {betweenness }}+\mathrm{SD}_{\text {betweenness }}$ was a hub gene. It was postulated that hub genes are essential for protein networks encoded by chemoresistance-related genes of ovarian cancer. To further confirm the reliability of the hub genes generated by the bioinformatics analysis, reverse transcription-quantitative PCR (RT-qPCR) was conducted to analyse the hub gene expression with respect to paclitaxel resistance.

Kaplan-Meier survival curve. Clinical data of patients with ovarian cancer were accessed from TCGA (https://cancergenome.nih.gov), which includes public genomic data. The Kaplan-Meier curve was generated using Kaplan-Meier plotter (https://kmplot.com/) and SPSS software (version 19.0; IBM Corp.). The log-rank and Tarone-Ware tests were used to determine statistical significance. Patients were grouped according to the quartile of the gene's FPKM value, with those before the first quartile as low expression and those after the third quartile as high expression. Kaplan-Meier survival curves were used to observe the effect of gene expression on the prognosis of different clinical groups. In addition, immunohistochemical data from patients with ovarian cancer were obtained from The Human Protein Atlas (HPA) database (https://www.proteinatlas.org).

Cell lines and RT-qPCR. The paclitaxel-sensitive human ovarian cancer cell line A2780 and the paclitaxel-resistant cell line A2780/PA were obtained from the BeNa Culture Collection (Beijing, China). All cell lines were cultured in RPMI-1640 medium (Gibco; Thermo Fisher Scientific, Inc.) supplemented with $10 \%$ FBS (Gibco; Thermo Fisher Scientific, Inc.), $50 \mathrm{U} / \mathrm{ml}$ penicillin and $50 \mathrm{U} / \mathrm{ml}$ streptomycin at $37^{\circ} \mathrm{C}$ in $5 \% \mathrm{CO}_{2}$. The medium of A2780/PA cells was supplemented with $800 \mathrm{ng} / \mathrm{ml}$ paclitaxel (Invitrogen; Thermo Fisher Scientific, Inc.) to maintain its drug-resistance phenotype.

Total RNA was extracted from A2780/PA and A2780 cells using TRIzol ${ }^{\circledR}$ (Invitrogen; Thermo Fisher Scientific Inc.). Then $2 \mu \mathrm{g}$ of total RNA was used to synthesise cDNA, using the High Capacity RNA-to-cDNA kit (TaqMan, Applied 
Biosystems). mRNA levels were examined using TransStart Top Green qPCR SuperMix (TransGen Biotech Co., Ltd). The relative expression level of each target gene was normalised to ACTB (internal control; Forward, 5'-CTTAGTTGCGTT ACACCCTTTCTTG-3' and reverse, 5'-CTGTCACCTTCA CCGTTCCAGTTT-3') using a $2^{-\Delta \Delta C q}$ relative quantification method $(21,22)$. All reactions were performed using the following cycling parameters: Initial denaturation at $94^{\circ} \mathrm{C}$ for $30 \mathrm{sec}$; followed by 45 cycles of $94^{\circ} \mathrm{C}$ for $5 \mathrm{sec}, 60^{\circ} \mathrm{C}$ for $15 \mathrm{sec}$ and $72^{\circ} \mathrm{C}$ for $10 \mathrm{sec}$. Primer sequences are provided on Table SI.

Measurement of reactive oxygen species (ROS). The oxidant-sensitive dye dichloro-dihydro-fluorescein diacetate (DCFH-DA; Beyotime Institute of Biotechnology) was used to detect intracellular ROS levels. Exponentially growing A2780 and A2780/PA cells were seeded in 6-well culture plates at a density of $3 \times 10^{5}$ cells/well. After $24 \mathrm{~h}$ of incubation, the fresh medium was changed and $400 \mathrm{ng} / \mathrm{ml}$ paclitaxel was added for $12 \mathrm{~h}$. Then the cells were trypsinized and incubated in $2 \mathrm{ml}$ DCFH-DA $(10 \mu \mathrm{M})$ working solution for $20 \mathrm{~min}$ at $37^{\circ} \mathrm{C}$ in the dark, washed with PBS twice and then evaluated using a guava easyCyte flow cytometer (Millipore). Data analysis was performed using ExpressPro software (version 8.1; Millipore).

Measurement of lipid peroxidation. The fluorescent lipid peroxidation reporter molecule C11-BODIPY (Beyotime Institute of Biotechnology) was detected lipid peroxidation. Exponentially growing A2780 and A2780/PA cells were seeded in 6-well culture plates at a density of $3 \times 10^{5}$ cells/well. After $24 \mathrm{~h}$ of incubation, the fresh medium was changed and $400 \mathrm{ng} / \mathrm{ml}$ paclitaxel was added for $12 \mathrm{~h}$. Then the cells were trypsinized and incubated in $2 \mathrm{ml} \mathrm{C} 11$-BODIPY $(10 \mu \mathrm{M})$ working solution for $20 \mathrm{~min}$ at $37^{\circ} \mathrm{C}$ in the dark, washed with PBS twice and then evaluated using a guava easyCyte flow cytometer (Millipore). Data analysis was performed using ExpressPro software.

Statistical analysis. Data are expressed as the mean \pm standard error of the mean of three independent experiments. Statistical analysis was performed using SPSS 19.0 software (IBM Corp.). Student's t-test was used to compare the mean values of two groups. $\mathrm{P}<0.05$ was considered to indicate a statistically significant difference.

\section{Results}

Data preprocessing. After GSE73935 dataset was preprocessed with multi-array average (RMA) (23) integrated algorithm in $\mathrm{R}$ software 'Bioconductor' package, the data was converted into logarithmic form. The original chip data and the RMA preprocessed data are shown in Fig. 1.

Characteristics of dataset and DEG identification. A total of 1,062 DEGs were identified from the GSE73935 dataset, of which 598 were upregulated and 464 were downregulated. A total of 188 DEGs were identified in cisplatin resistance/chemotherapy sensitivity, of which 126 were downregulated and 62 were upregulated (Fig. 2A; Table SII). A total of 98 DEGs were identified in the doxorubicin resistance/chemotherapy sensitivity group, of which 32 were downregulated and 66 were upregulated (Fig. 2B; Table SIII). In the paclitaxel resistant/sensitive group, 232 DEGs were identified, of which 101 were downregulated and 131 were upregulated (Fig. 2C; Table SIV). In addition, 194 DEGs were identified in the topotecan resistance/chemotherapy sensitivity group, of which 46 were downregulated and 148 were upregulated (Fig. 2D; Table SV). According to ovarian cancer treatment guidelines (24), overlapping genes for the two-drug combinations were defined, including cisplatin plus doxorubicin, cisplatin plus paclitaxel and cisplatin plus topotecan. The number of overlapping genes can be determined by Venn plot analysis. (Fig. 2E). As shown in Table I, 37 DEGs were identified between the cisplatin and doxorubicin groups, of which 27 were downregulated and 10 were upregulated; 65 DEGs were identified between the cisplatin and paclitaxel groups, of which 51 were downregulated and 14 were upregulated; 49 DEGs were identified between the cisplatin and topotecan groups, of which 31 were downregulated and 18 were upregulated.

GO function term and KEGG pathway enrichment analysis. GO analysis of DEGs was divided into three functional groups, including molecular function, biological processes and cell composition. GO function term enrichment and KEGG pathway enrichment were performed on upregulated and downregulated DEGs of the four separate sets of data. Significant results are shown in Table SVI. The top five descriptions of each part after the p-value is ranked from small to large (Fig. 3A-H).

GO function enrichment of the cisplatin resistance group demonstrated that the upregulated genes were mainly enriched in 'receptor-mediated endocytosis of virus by host cell' and 'secretory granule membrane system development', while the downregulated genes were mainly enriched in 'multicellular organismal process' and 'phenanthrene 9,10-monooxygenase activity'. In KEGG signaling pathways enrichment, the upregulated genes were mainly enriched in 'axon guidance' and 'non-small cell lung cancer', while the downregulated genes were mainly enriched in 'melanoma' and 'regulation of actin cytoskeleton' (Fig. 3A and B).

GO function enrichment of the doxorubicin resistance group demonstrated that the upregulated genes were mainly enriched in 'regulation of cell communication by electrical coupling' and 'cell-cell junction', while the downregulated genes were mainly enriched in 'regulation of multicellular organismal process', 'phosphatidylcholine-translocating ATPase activity' and 'plasma membrane part'. In KEGG signaling pathways enrichment, the upregulated genes were mainly enriched in 'chronic myeloid leukemia' and 'axon guidance', while the downregulated genes were mainly enriched in 'bile secretion' and 'pancreatic secretion' (Fig. 3C and D).

GO function enrichment of the paclitaxel resistance group demonstrated that the upregulated genes were mainly enriched in 'system development', 'sulfur compound binding' and 'proteinaceous extracellular matrix', while the downregulated genes were mainly enriched in 'cell adhesion' and 'cell junction'. In KEGG signaling pathways enrichment, the upregulated genes were mainly enriched in 'arrhythmogenic right ventricular cardiomyopathy (ARVC)' and 'glycine, serine and threonine metabolism', while the downregulated 

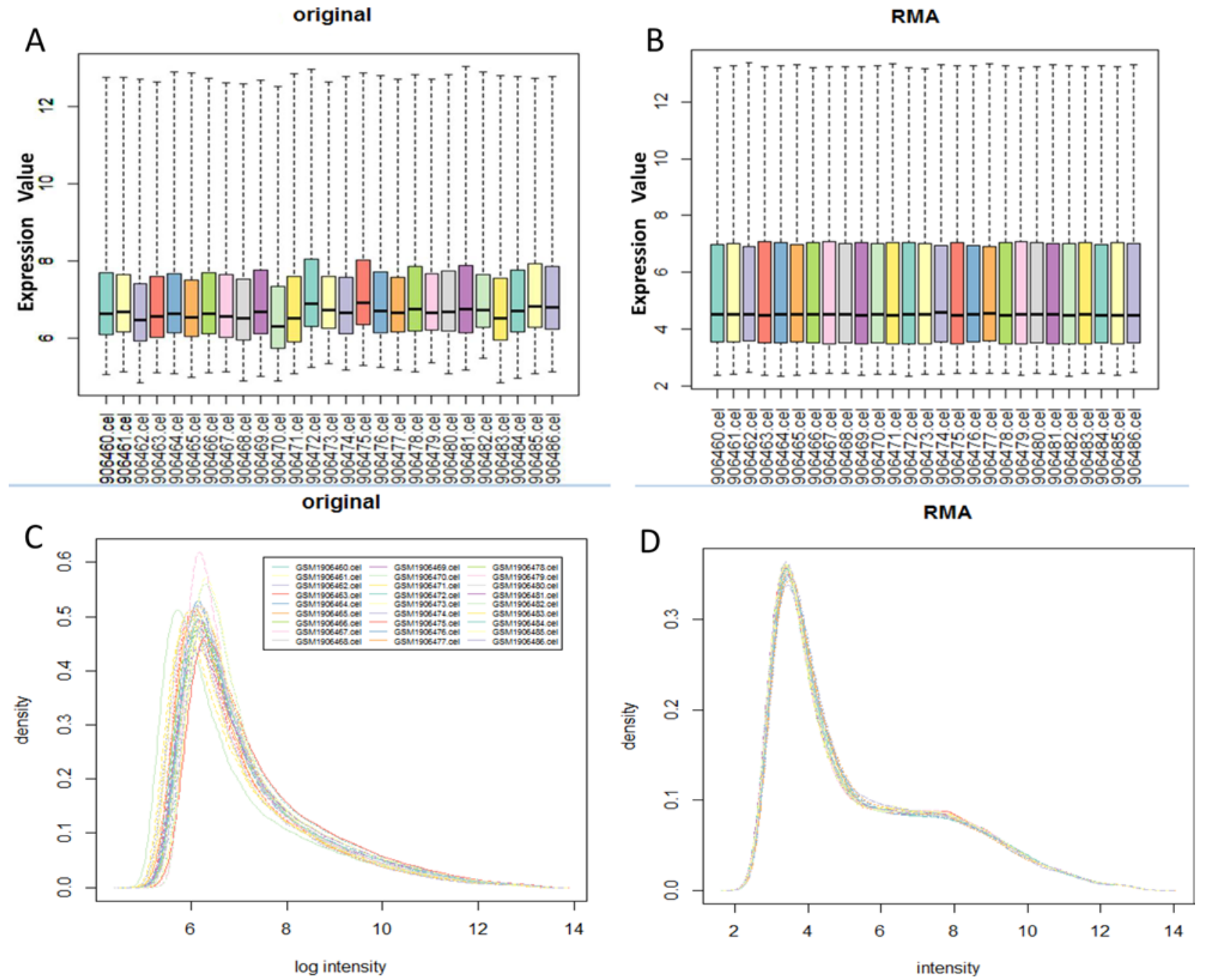

Figure 1. RMA preprocessing of the GSE73935 dataset. (A and B) Box plot after RMA processing. The median value of each sample is close. (C and D) Signal intensity distribution diagram after RMA processing. The curves of each sample coincide, and it is close to the Gaussian distribution, so the RMA method was selected. RMA, robust multi-array average.

genes were mainly enriched in 'VEGF signaling pathway' and 'Fc gamma R-mediated phagocytosis' (Fig. 3E and F).

$\mathrm{GO}$ function enrichment of the topotecan resistance group demonstrated that the upregulated genes were mainly enriched in 'system development', 'GPI-linked ephrin receptor activity' and 'integral component of plasma membrane', while the downregulated genes were mainly enriched in 'regulation of multicellular organismal process', 'receptor binding' and 'extracellular matrix'. In KEGG signaling pathways enrichment, the upregulated genes were mainly enriched in 'axon guidance' and 'Epithelial cell signaling in Helicobacter pylori', while the downregulated genes were mainly enriched in 'African trypanosomiasis' and 'Metabolism of xenobiotics by cytochrome P450' (Fig. 3G and H).

PPI network. According to results from STRING analysis, compared with the chemotherapy-sensitive group, 118 nodes and 77 edges in the cisplatin-resistant group met the screening conditions (score $>0.4$ ), and a PPI network map was drawn (Fig. 4A). The PPI network map of the doxorubicin-resistant group comprises 83 nodes and 52 edges (Fig. 4B). The PPI network map of paclitaxel resistance and chemotherapy sensitivity has 163 nodes and 170 edges (Fig. 4C), and the PPI network map of topotecan resistance and chemotherapy sensitivity has 173 nodes and 193 edges (Fig. 4D). In addition, the PPI network map of cisplatin plus doxorubicin has 11 nodes and seven edges (Fig. 4E), the PPI network map of cisplatin plus paclitaxel has 27 nodes and 22 edges (Fig. 4F), and the PPI network map of cisplatin plus topotecan has 16 nodes and 14 edges (Fig. 4G).

The hub genes were obtained by calculating the degree and betweenness parameters of the PPI network map. Among them, the cisplatin-resistant chemotherapy group has five hub genes, the doxorubicin-resistant chemotherapy group has five hub genes, the paclitaxel-resistant chemotherapy group has 15 hub genes and the topotecan-resistant chemotherapy group has five hub genes (Table II). The cisplatin plus doxorubicin group had three hub genes, the cisplatin plus paclitaxel group has six hub genes, and the cisplatin plus topotecan group has five hub genes (Table III).

Validation of hub genes and survival analysis. To verify the proposed clinical protocol, survival analyses were performed using data from patients with ovarian cancer in the TCGA 

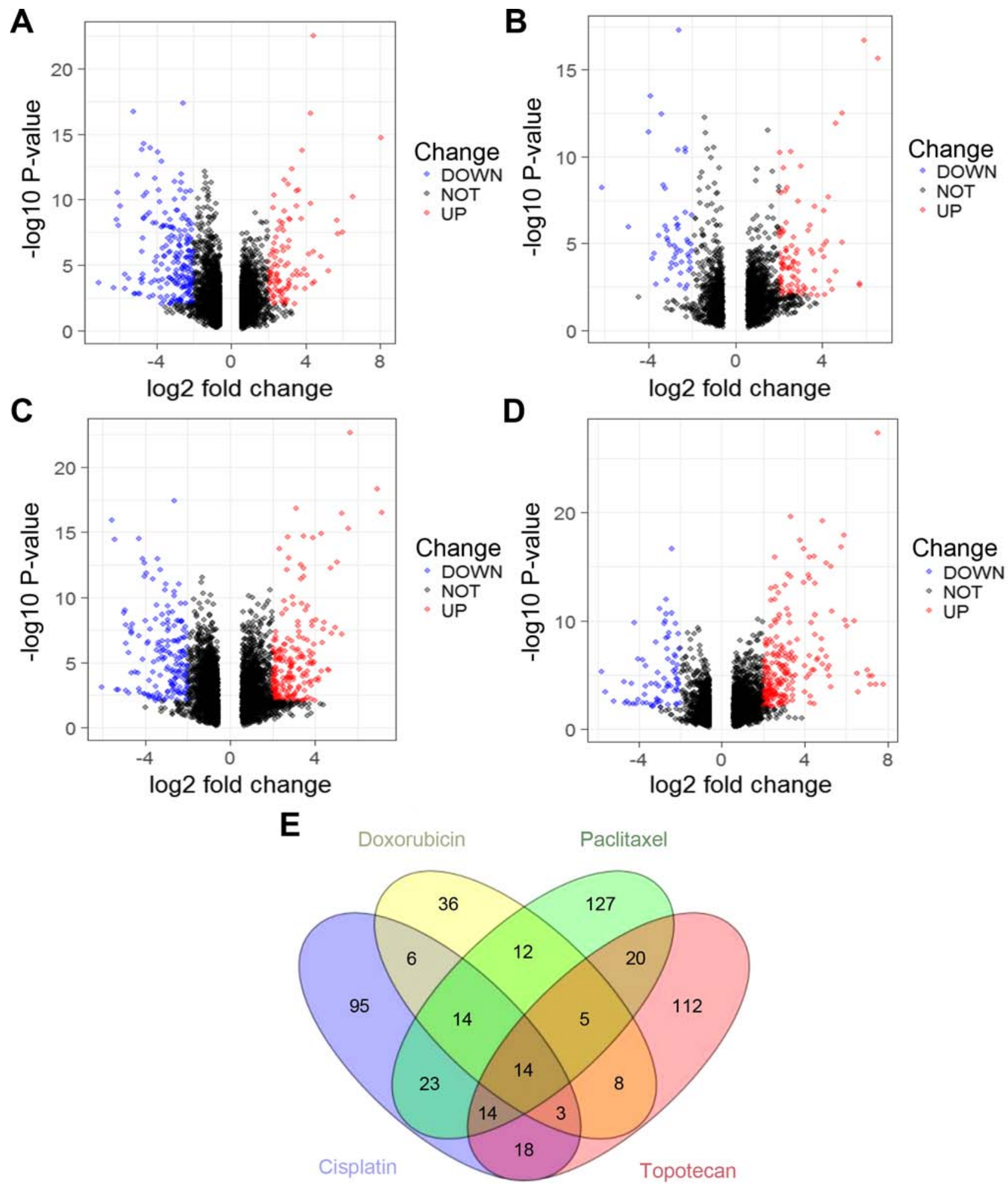

Figure 2. DEGs and overlapping DEGs between four sets of samples. (A) Cisplatin-resistance, (B) doxorubicin-resistance, (C) paclitaxel-resistance and (D) topotecan-resistance. The red and blue points represent upregulated and downregulated expression of genes screened on the basis of log $2 \mathrm{FCl}>2.0$ and $\mathrm{P}<0.01$, respectively. The black points represent genes with no significant difference in expression levels. (E) Venn diagram of overlapping DEGs in the four drug-resistant ovarian cancer groups. DEGs, differentially expressed genes.

database (Fig. 5). Considering the clinical medication information of TCGA ovarian cancer patients and cisplatin plus paclitaxel is the preferred chemotherapy regimen, survival analysis was grouped according to the expression of the hub gene in the cisplatin plus paclitaxel group and the observed prognosis in the cisplatin plus paclitaxel group and the other group, which mainly included sequential doxorubicin-cisplatin/paclitaxel and sequential topotecan-cisplatin/paclitaxel. The results demonstrated that the expression of epidermal growth factor-like repeats and discoidin I-like domains 3 (EDIL3), NRAS proto-oncogene (NRAS), hyaluronan and proteoglycan link protein 1 (HAPLNI) and activated protein $\mathrm{C}$ 
Table I. Combination of two chemotherapy drugs resistance group overlapped DEGs.

Group

Number of overlapping DEGs

(downregulate; upregulate)

Exclusive overlapping DEG ratio $(\%)^{\mathrm{a}}$

$\begin{array}{ll}\text { Cisplatin + doxorubicin } & 37(27 ; 10) \\ \text { Cisplatin + paclitaxel } & 65(51 ; 14) \\ \text { Cisplatin + topotecan } & 49(31 ; 18)\end{array}$

$16.2(6 / 37)$

$35.4(23 / 65)$

$36.7(18 / 49)$

${ }^{a}$ Exclusive overlapping gene ratio $(\%)=$ differential gene that is only resistant to two types of chemotherapeutic drugs/different genes that are resistant to two types of chemotherapeutic drugs. DEG, differentially expressed genes.

A

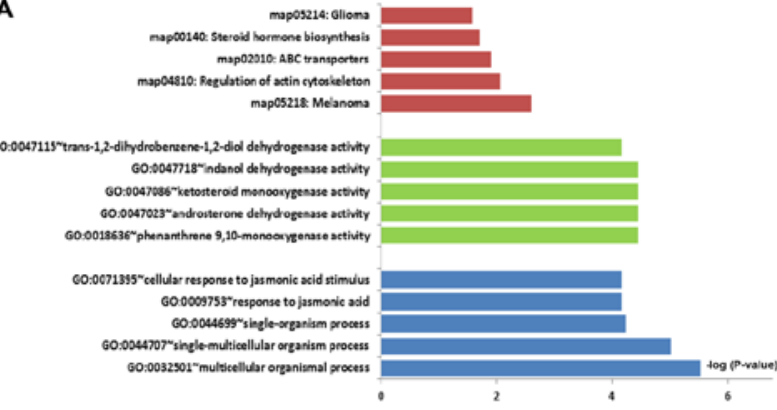

C
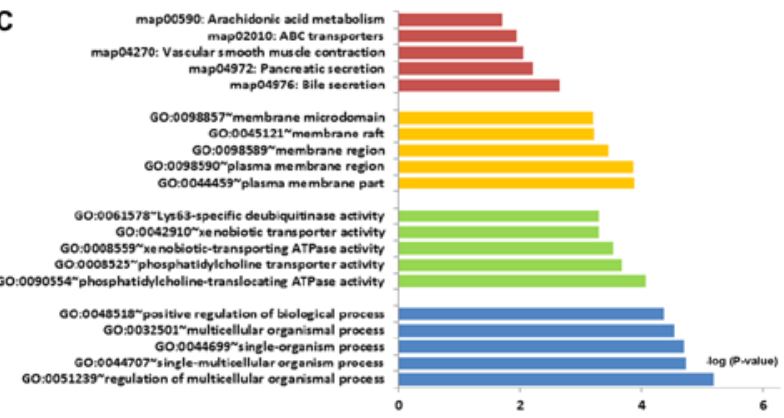

E
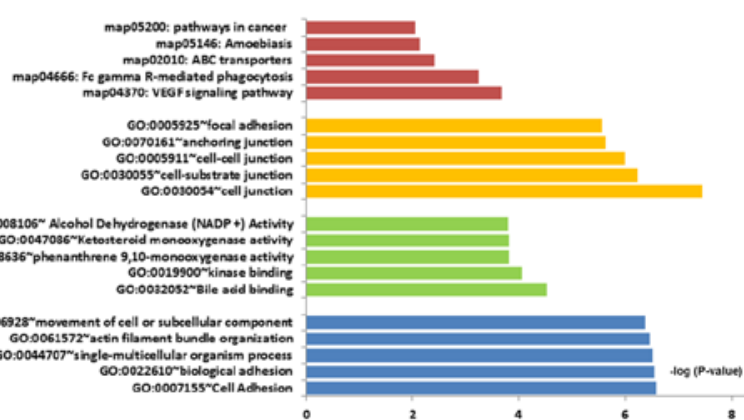

G
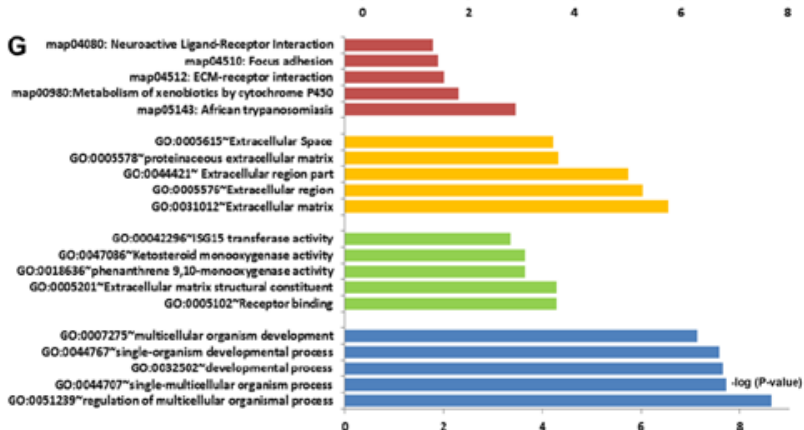

B

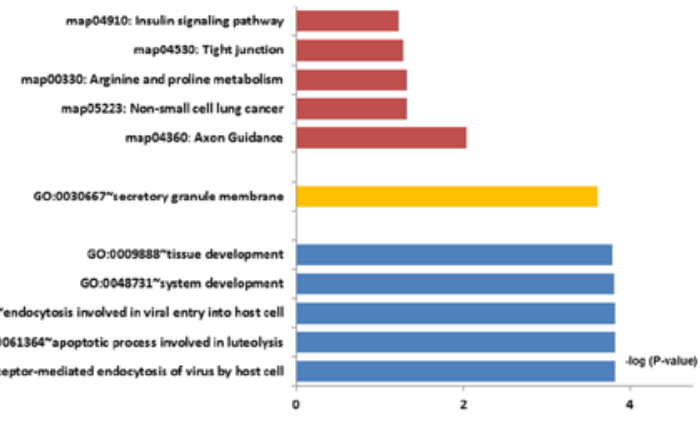

D

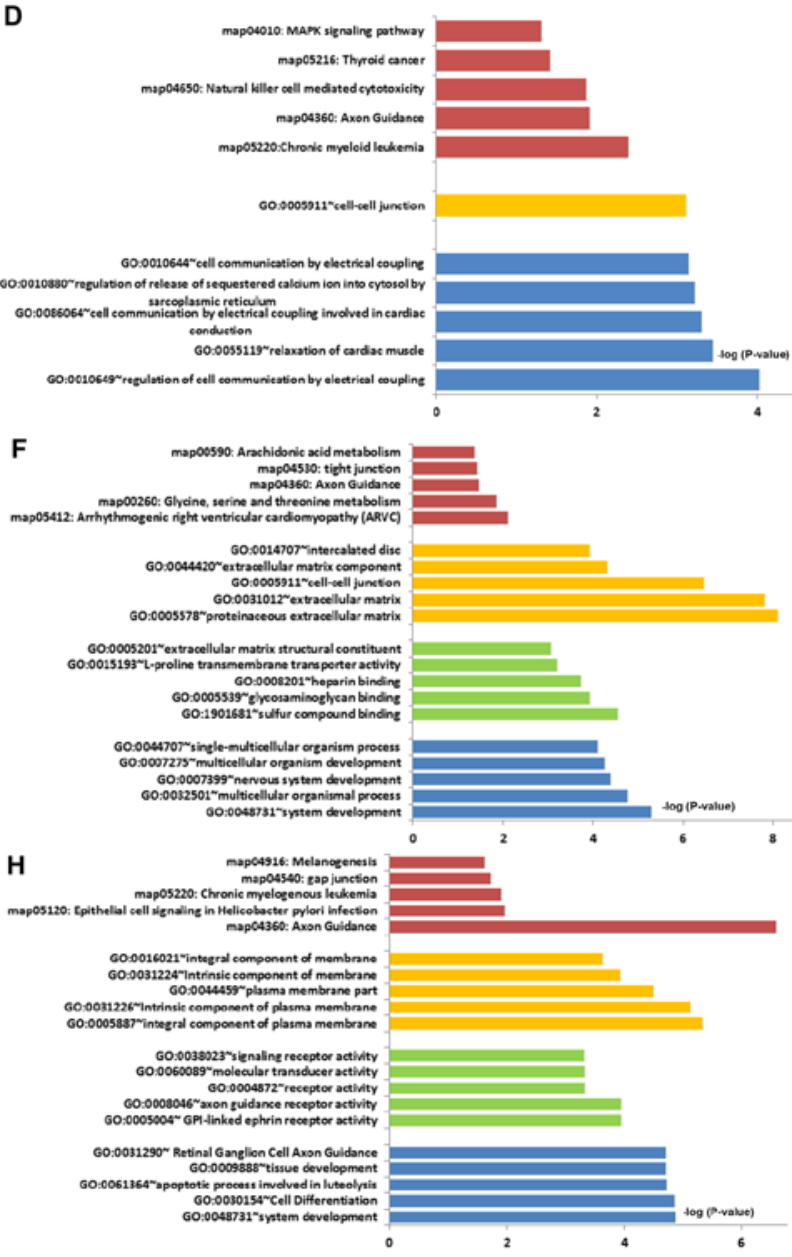

Figure 3. GO functional term and KEGG pathway enrichment. (A) Cisplatin resistance; upregulated DEGs. (B) Cisplatin resistance; downregulated DEGs. (C) Doxorubicin resistance; upregulated DEGs. (D) Doxorubicin resistance; downregulated DEGs. (E) Paclitaxel resistance; upregulated DEGs. (F) Paclitaxel resistance; downregulated DEGs. (G) Topotecan resistance; upregulated DEGs. (H) Topotecan resistance; downregulated DEGs. The red bars represent signalling pathways identified with the KEGG, The yellow, green and blue bars represent cell components, molecular function and biological process, respectively, identified with GO enrichment analysis. ABC, ATP-binding cassette; DEGs, differentially expressed genes; ECM, extracellular matrix; GO, Gene Ontology; KEGG, Kyoto Encyclopedia of Genes and Genomes. 
A

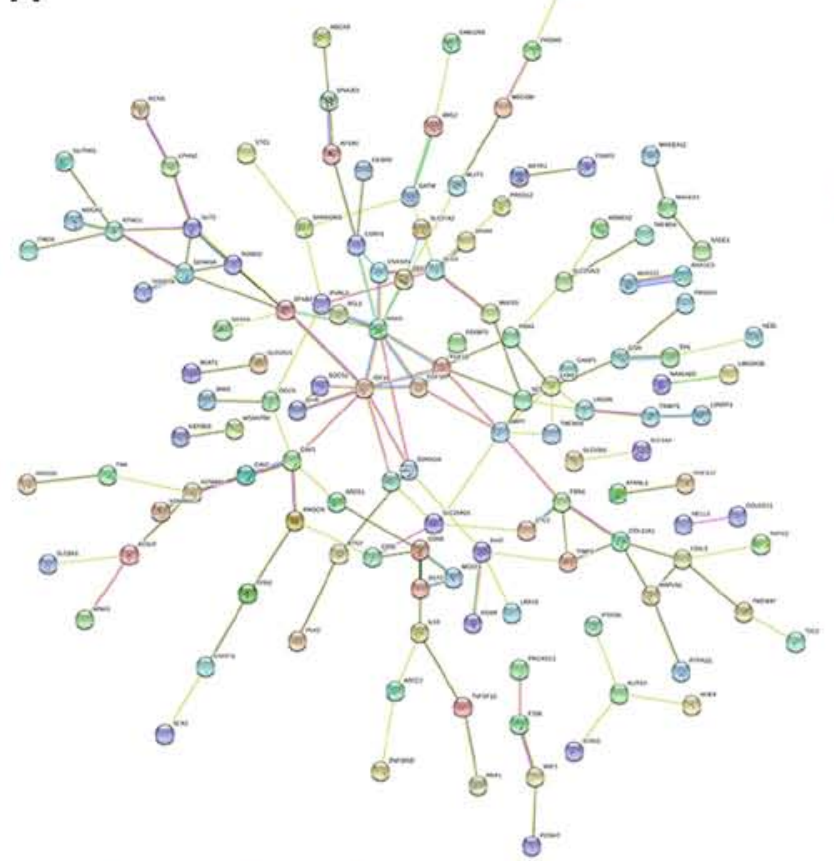

C
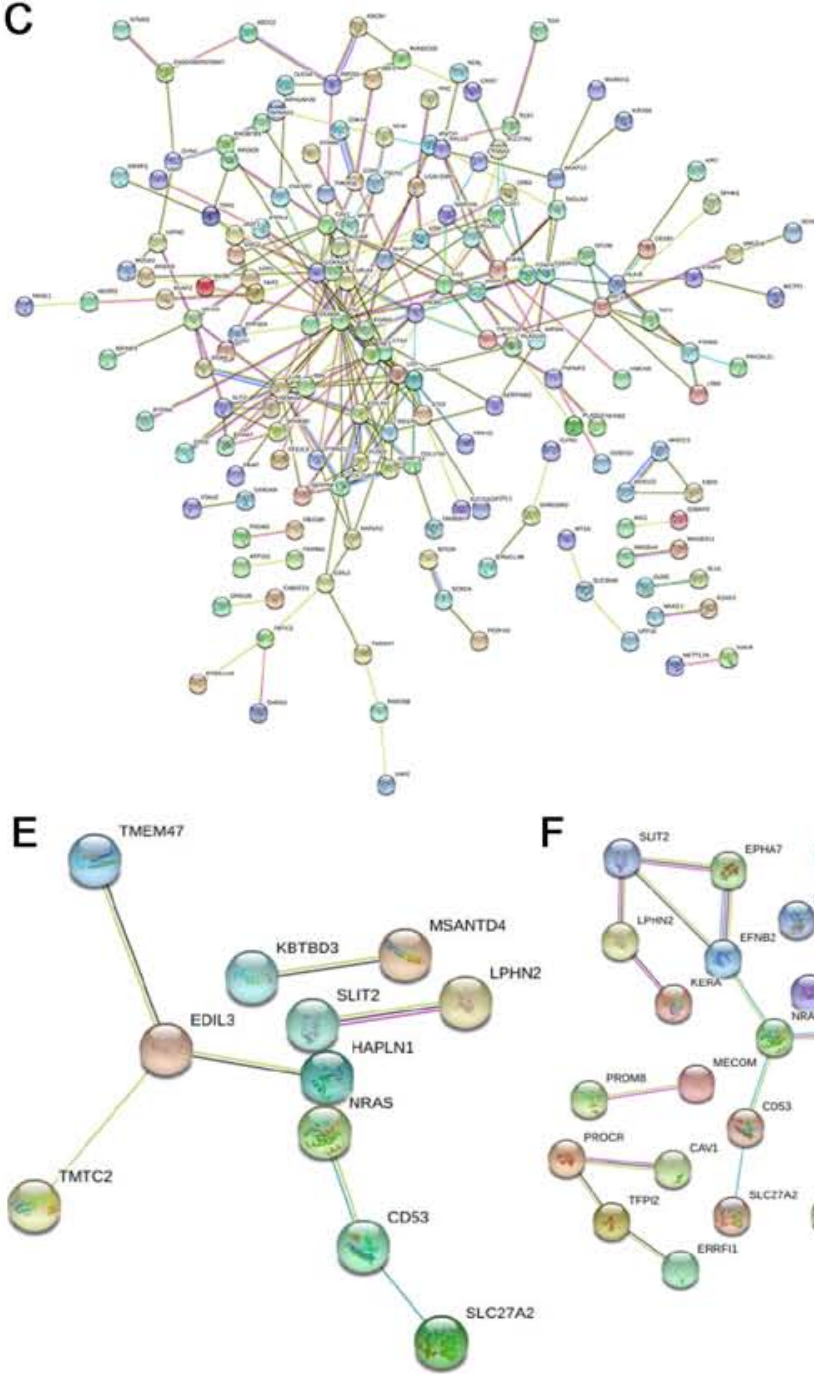

B

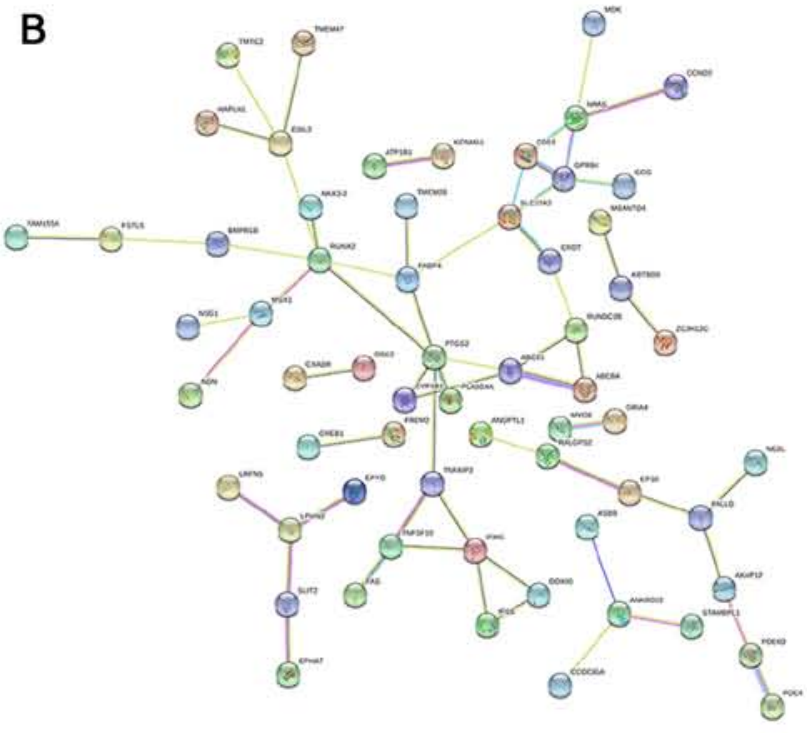

$\mathrm{F}$

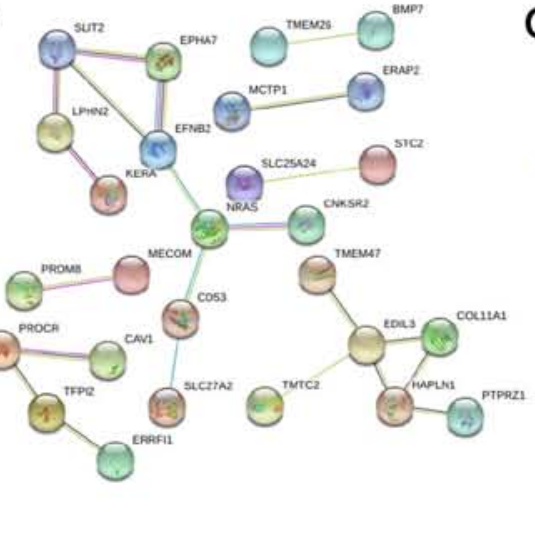

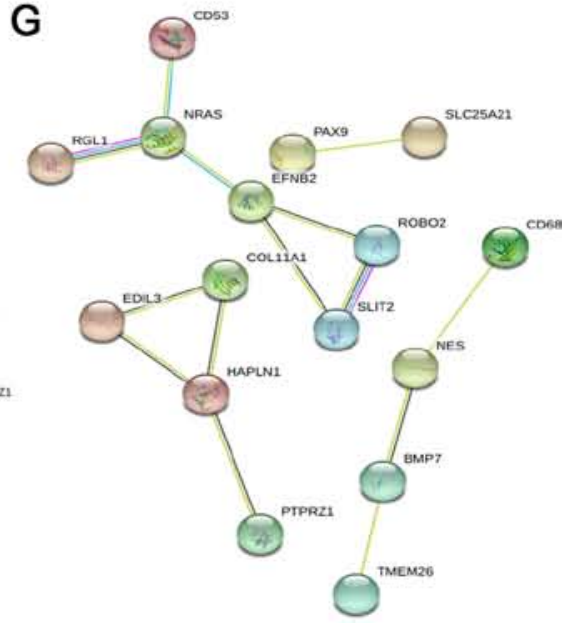

Figure 4. PPI networks of the identified DEGs for different drug conditions. (A) Cisplatin. (B) Doxorubicin. (C) Paclitaxel. (D) Topotecan. (E) Cisplatin plus doxorubicin. (F) Cisplatin plus paclitaxel. (G) Cisplatin plus topotecan. Circles represent genes, lines represent the interaction of proteins between genes, and the results within the circle represent the structure of proteins. Line color represents evidence of the interaction between the proteins. PPI, Protein-protein interaction; DEGs, differentially expressed genes. 
Table II. Single chemotherapy drug resistance group hub genes.

\begin{tabular}{|c|c|c|c|c|}
\hline Group & Hub gene & Degree & Betweenness & Expression \\
\hline \multirow[t]{5}{*}{ Cisplatin } & $I G F 1 R$ & 10 & $1,714.33$ & $\mathrm{Up}$ \\
\hline & NRAS & 9 & 830.33 & Down \\
\hline & FGF10 & 5 & 662.00 & $\mathrm{Up}$ \\
\hline & $D L G 3$ & 6 & 531.33 & Down \\
\hline & $C A V 1$ & 6 & 524.67 & Down \\
\hline \multirow[t]{5}{*}{ Doxorubicin } & $N R A S$ & 10 & 799.67 & Down \\
\hline & $R U N X 2$ & 6 & 482.00 & Up \\
\hline & $M D K$ & 5 & 340.67 & Down \\
\hline & PTGS2 & 4 & 187.00 & $\mathrm{Up}$ \\
\hline & $M S X 1$ & 5 & 186.00 & Up \\
\hline \multirow[t]{15}{*}{ Paclitaxel } & $C D K N 2 A$ & 10 & $2,889.06$ & Up \\
\hline & $D L G 1$ & 11 & $2,437.20$ & Down \\
\hline & $C T G F$ & 9 & $2,173.22$ & Up \\
\hline & $M E T$ & 8 & $1,852.22$ & Up \\
\hline & $M A F$ & 6 & $1,741.90$ & Down \\
\hline & $F G F R 3$ & 6 & $1,721.56$ & Up \\
\hline & PIK3R1 & 5 & $1,703.86$ & Up \\
\hline & $F A S$ & 6 & $1,378.57$ & $\mathrm{Up}$ \\
\hline & $P T A F R$ & 5 & $1,326.63$ & Up \\
\hline & $A B C B 1$ & 7 & $1,276.65$ & Up \\
\hline & TNFAIP3 & 5 & $1,076.96$ & Up \\
\hline & $C D K 14$ & 8 & $1,042.03$ & Up \\
\hline & $T U B B 3$ & 5 & $1,034.81$ & Up \\
\hline & COL1A2 & 10 & 959.60 & Up \\
\hline & RHOBTBI & 6 & 899.87 & Down \\
\hline \multirow[t]{5}{*}{ Topotecan } & $N R A S$ & 16 & $4,558.12$ & Down \\
\hline & ACTA2 & 10 & $2,192.64$ & Up \\
\hline & $S P P 1$ & 9 & $2,111.47$ & Up \\
\hline & PRKG1 & 9 & $1,354.34$ & Up \\
\hline & $B M P 7$ & 8 & $1,694.94$ & Up \\
\hline
\end{tabular}

The hub genes need to satisfy both degree $\geq$ mean $_{\text {degree }}+$ standard deviation $\left(\mathrm{SD}_{\text {degree }}\right)$ and betweenness $\geq$ mean $_{\text {betweenness }}+\mathrm{SD}_{\text {betweenness }} A B C B 1$, ATP binding cassette subfamily B member $1 ; A C T A 2$, actin, alpha 2; BMP7, Bone morphogenetic protein 7; $C A V 1$, caveolin 1; $C D K 14$, cyclin dependent kinase 14; $C D K N 2 A$, cyclin dependent kinase inhibitor 2A; COL1A2, collagen type I alpha 2 chain; $C T G F$, connective tissue growth factor; $D L G 1$, disks large homolog 1; DLG3, disks large homolog 3; FAS, Fas cell surface death receptor; FGF10, fibroblast growth factor 10; FGFR3, fibroblast growth factor receptor 3; IGFIR, Insulin-like growth factor I receptor; MAF, MAF bZIP transcription factor; $M D K$, midkine; MET, MET proto-oncogene, receptor tyrosine kinase; MSX1, msh homeobox 1; NRAS, NRAS proto-oncogene; PIK3R1, phosphoinositide-3-kinase regulatory subunit 1; PRKG1, protein kinase cGMP-dependent 1; PTAFR, platelet activating factor receptor; $P T G S 2$, prostaglandin-endoperoxide synthase 2; RHOBTB1, Rho related BTB domain containing 1; RUNX2, RUNX family transcription factor 2; SPP1, secreted phosphoprotein 1; TNFAIP3, TNF alpha induced protein 3; TUBB3, tubulin beta 3 class III.

receptor $(P R O C R)$ were closely related to the prognosis of the cisplatin plus paclitaxel group. The expression of ephrin B2 (EFNB2) and tissue factor pathway inhibitor 2 (TFPI2) did not affect the prognosis of cisplatin plus paclitaxel group (Fig. S1).

In the cisplatin plus paclitaxel group, the five-year survival rate of the EDIL3 high expression group was $32 \%$, and the five-year survival rate of the EDIL3 low expression group was $50 \%$. Compared with the low expression group of EDIL3, the prognosis of patients with ovarian cancer in the high expression group of EDIL3 was worse $(\mathrm{P}<0.05$; Fig. $5 \mathrm{~A})$. In the other group, the expression of this gene was not associated with prognosis ( $\mathrm{P}>0.05$; Fig. 5B).
In the cisplatin plus paclitaxel group, the five-year survival rate of ovarian cancer patients with high NRAS expression was $38 \%$, and the five-year survival rate of patients with ovarian cancer with low NRAS expression was $23 \%$. Compared with the high expression group of NRAS, the prognosis of ovarian cancer patients in the low expression group of $N R A S$ was worse $(\mathrm{P}<0.05$; Fig. $5 \mathrm{C})$. In the other group, the expression of this gene was not associated with prognosis ( $P>0.05$; Fig. 5D).

In the cisplatin plus paclitaxel group, the five-year survival rate of patients with ovarian cancer with high expression of HAPLN1 was $28 \%$, and the five-year survival rate of patients with ovarian cancer with low expression of $H A P L N 1$ was $42 \%$. 
Table III. Combination of two chemotherapy drugs resistance group hub genes.

\begin{tabular}{|c|c|c|c|c|}
\hline Group & Hub gene & Degree & Betweenness & Expression \\
\hline \multirow[t]{3}{*}{ Cisplatin + doxorubicin } & EDIL3 & 3 & 6 & Up \\
\hline & $C D 53$ & 2 & 2 & Down \\
\hline & SLIT2 & 1 & 2 & Down \\
\hline \multirow[t]{6}{*}{ Cisplatin + paclitaxel } & EDIL3 & 4 & 14 & Up \\
\hline & $N R A S$ & 3 & 14 & Down \\
\hline & $E F N B 2$ & 3 & 12 & Down \\
\hline & HAPLN1 & 3 & 8 & Up (down) \\
\hline & TFPI2 & 2 & 4 & Down \\
\hline & PROCR & 2 & 4 & $\mathrm{Up}$ \\
\hline \multirow[t]{5}{*}{ Cisplatin + topotecan } & HAPLN1 & 3 & 4 & Up \\
\hline & NRAS & 3 & 4 & Down \\
\hline & EFNB2 & 3 & 4 & Down \\
\hline & $\mathrm{ROBO} 2$ & 2 & 2 & Down \\
\hline & COL11A1 & 2 & 2 & Down \\
\hline
\end{tabular}

The hub genes need to satisfy both degree $\geq$ mean $_{\text {degree }}+$ standard deviation $\left(\mathrm{SD}_{\text {degree }}\right)$ and betweenness $\geq$ mean $_{\text {betweenness }}+\mathrm{SD}_{\text {betweenness }} \mathrm{COL} 11 \mathrm{~A} 1$, collagen type $11 \alpha 1$ chain; EDIL3, epidermal growth factor-like repeats and discoidin I-like domains 3; EFNB2, ephrin B2; HAPLN1, hyaluronan and proteoglycan link protein 1; NRAS, NRAS proto-oncogene; PROCR, activated protein C receptor; ROBO2, roundabout guidance receptor 2; SLIT2, slit guidance ligand 2; TFPI2, tissue factor pathway inhibitor 2.
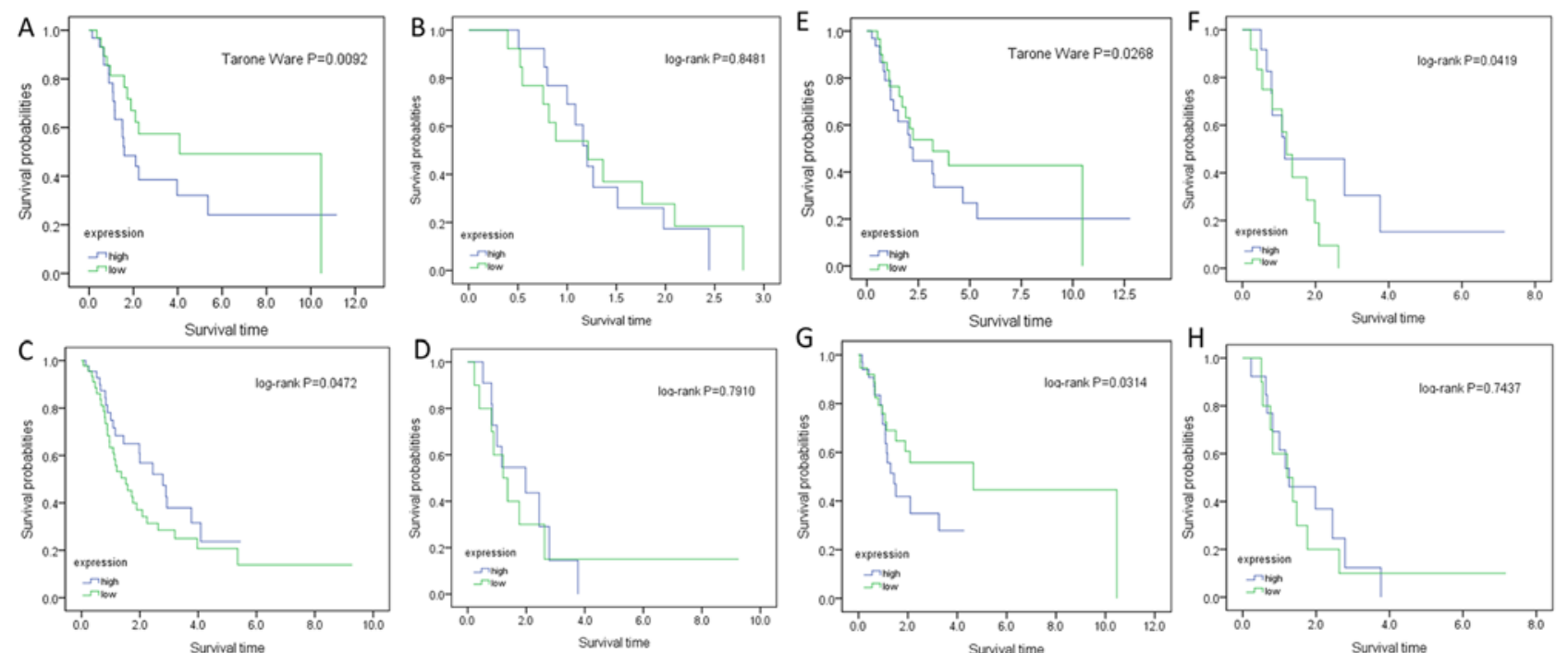

Figure 5. Kaplan-Meier survival curves of patients with ovarian cancer, stratified by gene expression levels. (A) EDIL3, cisplatin plus paclitaxel. (B) EDIL3, others. (C) NRAS, cisplatin plus paclitaxel. (D) NRAS, others. (E) HAPLN1, cisplatin plus paclitaxel. (F) HAPLN1, others. (G) PROCR, cisplatin plus paclitaxel. (H) PROCR, others. others, sequential doxorubicin-cisplatin/paclitaxel and sequential topotecan-cisplatin/paclitaxel. EDIL3, epidermal growth factor-like repeats and discoidin I-like domains 3; HAPLN1, hyaluronan and proteoglycan link protein 1; NRAS, NRAS proto-oncogene; PROCR, activated protein $\mathrm{C}$ receptor.

Compared with the low expression group of $H A P L N 1$, the prognosis of patients with ovarian cancer in the high expression group of $H A P L N 1$ was worse $(\mathrm{P}<0.05$; Fig. 5E). In the other group, the survival rate of patients with ovarian cancer with low expression of HAPLN1 decreased to 0 at 2.7 years, while the five-year survival rate of patients with ovarian cancer with high expression of HAPLN1 was $48 \%$, and the prognosis of the low expression group was worse $(\mathrm{P}<0.05$; Fig. $5 \mathrm{~F})$.

In the cisplatin plus paclitaxel group, the five-year survival rate of patients with ovarian cancer with high PROCR expression was $30 \%$, and the five-year survival rate of patients with ovarian cancer with low PROCR expression was $48 \%$.
Compared with the low expression group of PROCR, the prognosis of patients with ovarian cancer in the high expression group of $P R O C R$ was worse $(\mathrm{P}<0.05$; Fig. $5 \mathrm{G})$. In the other group, the expression of this gene was not associated with prognosis $(\mathrm{P}>0.05$; Fig. $5 \mathrm{H})$.

In addition, since TCGA data do not contain clinical information of other drug groups, which included cisplatin, paclitaxel, cisplatin + doxorubicin and cisplatin + topotecan, to verify that the hub genes of the other drug groups are also suitable for clinical samples, the immunohistochemistry results for patients with ovarian cancer in HPA database were used. The Insulin-like growth factor I receptor $(I G F 1 R)$ in the cisplatin 
A

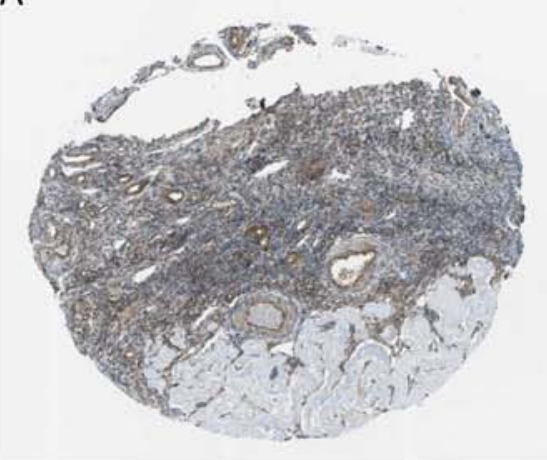

D

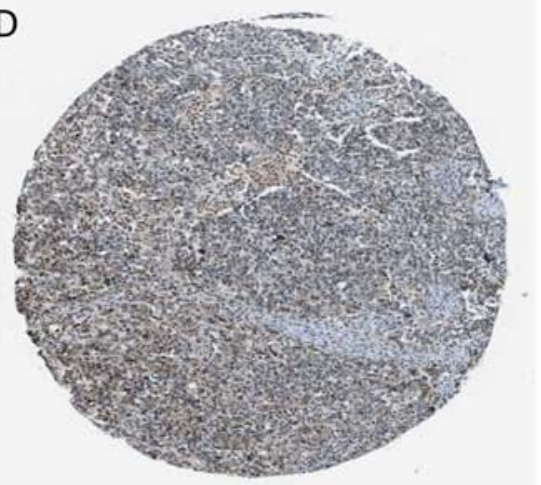

G

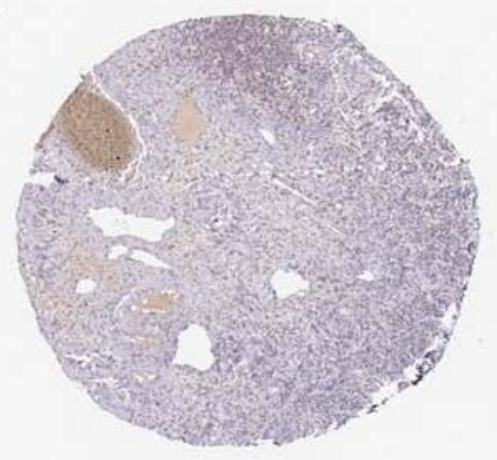

J

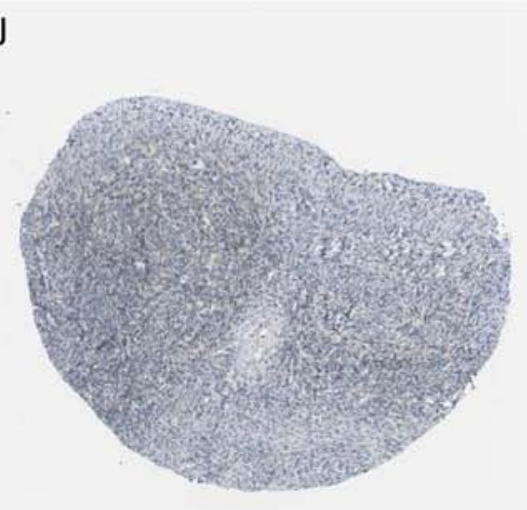

B

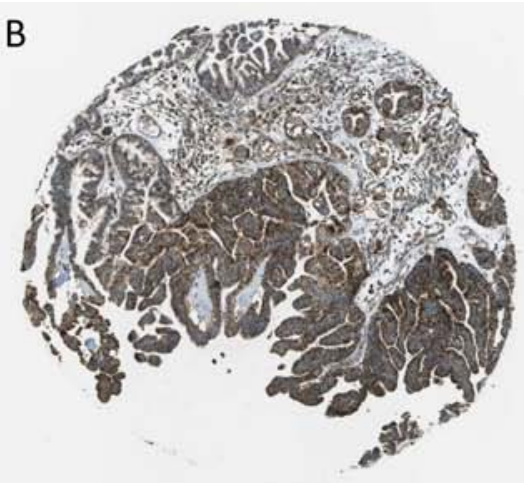

E

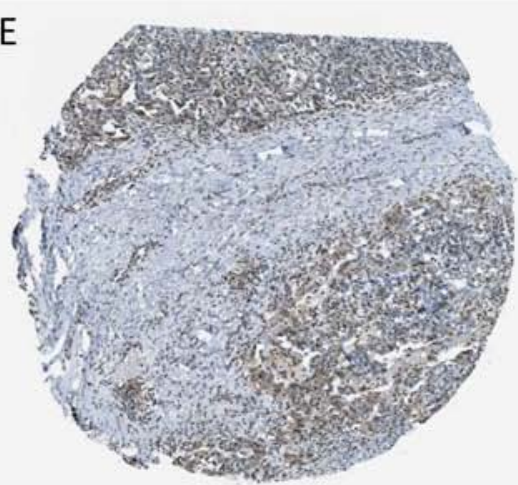

H

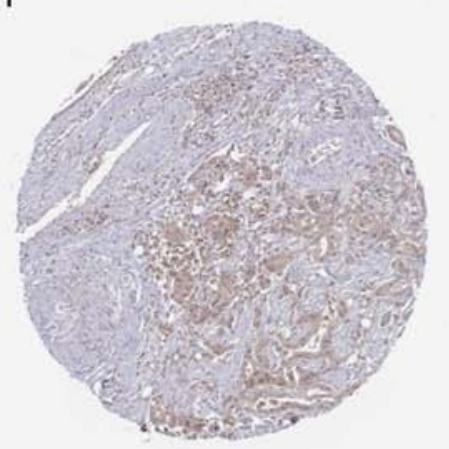

$\mathrm{K}$

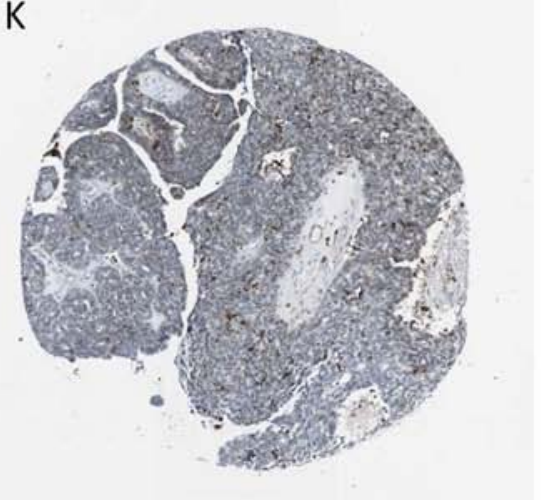

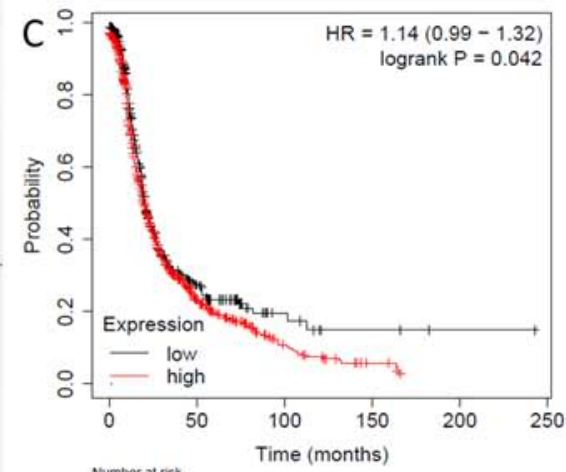

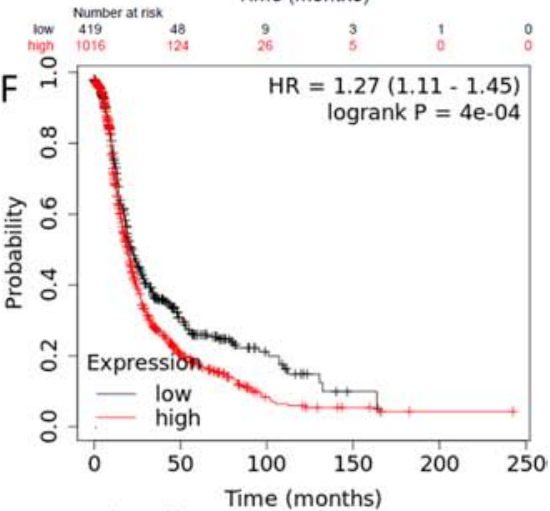

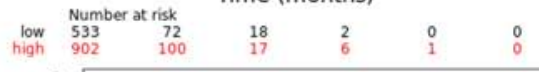
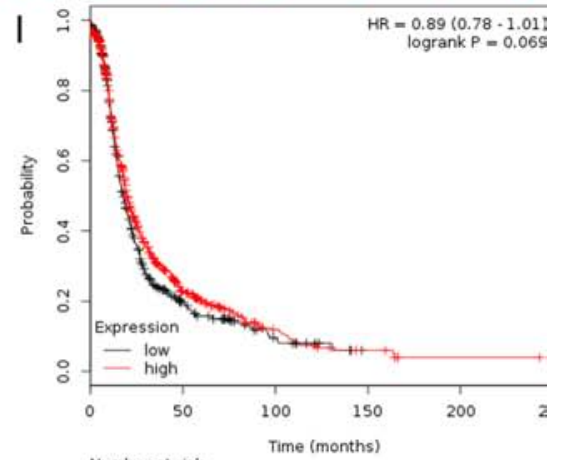

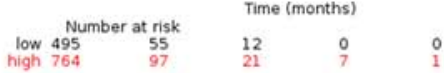

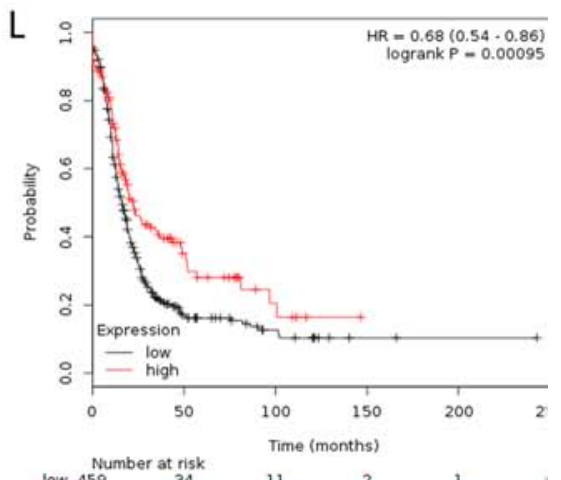

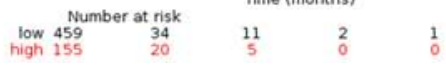

Figure 6. Immunohistochemistry and Kaplan-Meier survival curves of patients with ovarian cancer, stratified by gene expression levels. (A) IGF1R normal. (B) IGF1R ovarian cancer. (C) IGF1R survival curve. (D) CDKN2A normal. (E) CDKN2A ovarian cancer. (F) CDKN2A survival curve. (G) CD53 normal. (H) CD53 ovarian cancer. (I) CD53 survival curve. (J) ROBO2 normal. (K) ROBO2 ovarian cancer. (L) ROBO2 survival curve. IGF1R, insulin-like growth factor I receptor; $\mathrm{ROBO}$, roundabout guidance receptor 2; CDKN2A, cyclin-dependent kinase inhibitor 2A; Source: Human Protein Atlas.

group, cyclin-dependent kinase inhibitor $2 \mathrm{~A}(C D K N 2 A)$ in the paclitaxel group, $C D 53$ in the cisplatin plus doxorubicin group, and Roundabout guidance receptor 2 (ROBO2) in the cisplatin plus topotecan group were verified, and the effect of the expression of each hub gene on prognosis was observed with the Kaplan-Meier Plotter database.

$I G F 1 R$ is a hub gene that was upregulated in ovarian cancer cisplatin-resistant cells and not in other drug-resistant cell 


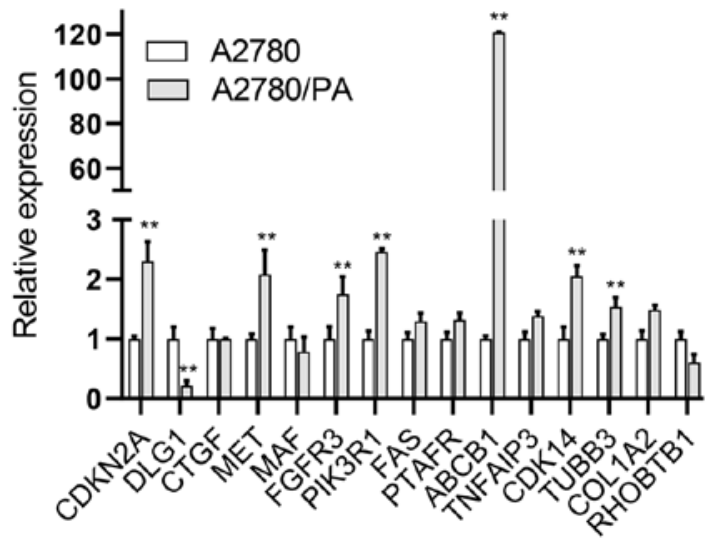

Figure 7. mRNA expression of hub genes in A2780/PA and A2780 cell lines. ${ }^{* *} \mathrm{P}<0.01$ vs. A2780. ABCB1, ATP binding cassette subfamily B member 1; CDKN2A, cyclin dependent kinase inhibitor 2A; CDK14, cyclin dependent kinase 14; COL1A2, collagen type I alpha 2 chain; CTGF, connective tissue growth factor; DLG1, disks large homolog 1; FAS, Fas cell surface death receptor; FGFR3, fibroblast growth factor receptor 3; MAF, MAF bZIP transcription factor; MET, MET proto-oncogene, receptor tyrosine kinase; PIK3R1, phosphoinositide-3-kinase regulatory subunit 1; PTAFR, platelet activating factor receptor; RHOBTB1, Rho related BTB domain containing 1 ; TNFAIP3, TNF alpha induced protein 3; TUBB3, tubulin beta 3 class III.

lines. It showed medium positive protein expression in normal ovarian tissues (Fig. 6A) and high positive protein expression in ovarian cancer tissues (Fig. 6B). IGF1R was significantly upregulated in tumor tissues compared with normal tissues. The five-year survival rate of patients with ovarian cancer with high $I G F I R$ expression was $15 \%$, and the five-year survival rate of patients with ovarian cancer with low IGFIR expression was $27 \%$. Compared with the low expression group of $I G F 1 R$, the prognosis of patients with ovarian cancer with high expression of $I G F I R$ was worse $(\mathrm{P}<0.05$; Fig. 6C).

$C D K N 2 A$ was identified as a hub gene that was upregulated in paclitaxel-resistant ovarian cancer cells but not in other drug-resistant cell lines. It showed low positive protein expression in normal ovarian tissue (Fig. 6D) and high positive protein expression in ovarian cancer tissues (Fig. 6E). CDKN2A was significantly upregulated in tumor tissues compared with normal tissues. The 5 -year survival rate of patients with ovarian cancer with high $C D K N 2 A$ expression was $18 \%$, and the 5-year survival rate of patients with ovarian cancer with low $C D K N 2 A$ expression was $28 \%$. The prognosis of patients with ovarian cancer, with high expression of $C D K N 2 A$ was worse compared with the low expression group of $C D K N 2 A$ $(\mathrm{P}<0.01$; Fig. 6F).

CD53 was identified as a hub gene with downregulated in the cisplatin plus doxorubicin group. It was found that this gene showed medium positive protein expression in normal ovarian tissue (Fig. 6G) and low positive protein expression in ovarian cancer tissues (Fig. 6H). CD53 was significantly downregulated in tumor tissues compared with normal tissues. CD53 expression had no significant effect on the five-year survival rate of patients with ovarian cancer ( $\mathrm{P}>0.05)$ (Fig. 6I).

$R O B O 2$ is a hub gene with downregulated in the cisplatin plus topotecan group. This gene showed low positive protein expression in normal ovarian tissues (Fig. 6J) and no protein expression was observed in ovarian cancer tissues (Fig. 6K). $\mathrm{ROBO} 2$ was significantly downregulated in tumor tissues compared with normal tissues. The five-year survival rate of patients with ovarian cancer with high $R O B O 2$ expression was $30 \%$, whereas that of patients with low $R O B O 2$ expression was $18 \%$. Compared with the high $R O B O 2$ expression group, the prognosis of patients with ovarian cancer in the low ROBO2 expression group was worse $(\mathrm{P}<0.01$; Fig. $6 \mathrm{~L})$.

Evaluation of hub gene expression in ovarian cancer. To verify the expression of hub genes in the paclitaxel-resistant and sensitive network, A2780/PA and A2780 cell lines were used, respectively. Relative mRNA expression levels of hub genes in A2780/PA and A2780 cells were quantified by qPCR, and the results showed that the average mRNA expression levels of disks large homolog 1 (DLGl) was significantly lower in A2780/PA cells compared with expression levels in A2780 cells. Furthermore, the average mRNA expression levels of ATP binding cassette subfamily B member 1 ( $A B C B 1), C D K N 2 A$, cyclin dependent kinase 14 (CDK14), fibroblast growth factor receptor 3 (FGFR3), MET proto-oncogene, receptor tyrosine kinase $(M E T)$, phosphoinositide-3-kinase regulatory subunit 1 (PIK3RI) and tubulin beta 3 class III (TUBB3) were significantly higher in A2780/PA cells compared to A2780 cells (Fig. 7).

Effect of ROS level on paclitaxel resistance in ovarian cancer. ROS serve a role in cellular responses to stress and are associated with apoptosis through mitochondrial DNA damage which has an effect on drug resistance (25). The role of mitochondrial ROS-mediated mechanisms, along with ROS prominent roles and modulation of metabolic events may be essential contributors for cancer drug resistance (26). Recent observations demonstrate that chronic and abnormally high ROS levels may instigate or accentuate cancer phenotypes, including drug resistance $(27,28)$. Therefore, intracellular ROS levels were measured by flow cytometry analysis after paclitaxel treatment. The results indicated that A2780/PA cells had significantly lower levels of ROS compared with A2780 cells after paclitaxel treatment (Fig. 8A-D).

Effect of lipid peroxidation on paclitaxel resistance in ovarian cancer. Elevated ROS can cause apoptosis by increasing the level of cellular lipid peroxidation and consequently augmenting the permeability of the mitochondrial membrane (29). Thus, intracellular lipid peroxidation levels were measured by flow cytometry analysis after paclitaxel treatment. The results indicated that A2780/PA cells had significantly lower lipid peroxidation than A2780 cells after paclitaxel treatment (Fig. 8E-H).

From the above results, it can be seen that the ROS level of A2780/PA was significantly lower than that of A2780 after paclitaxel administration, and the degree of lipid peroxidation was also significantly reduced, indicating that resistant cell lines can withstand ROS and lipid oxidation to enhance drug resistance. In addition, it was verified that the expression of the hub gene of each cell group was consistent with the gene expression of the clinical samples of ovarian cancer.

\section{Discussion}

Chemoresistance in ovarian cancer has been a hot topic in recent years. In the present study, bioinformatics analyses 
A

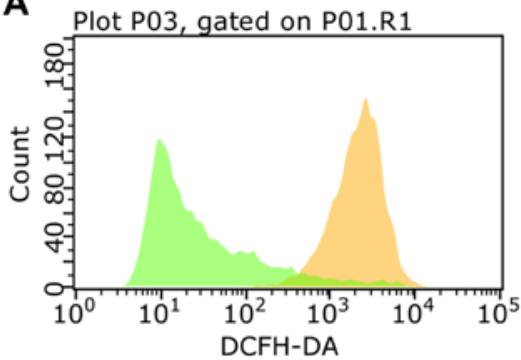

C

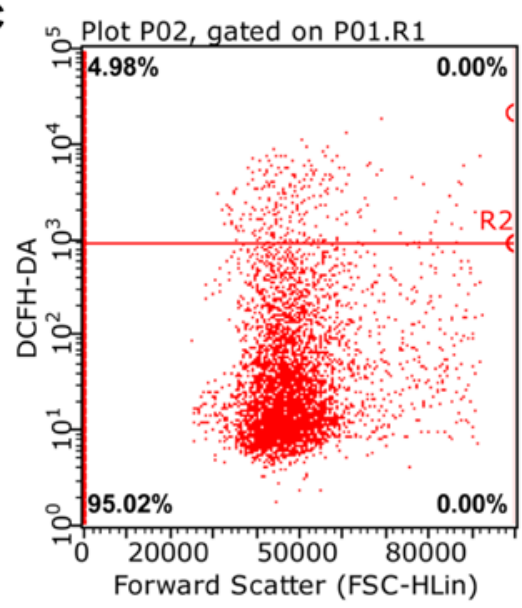

E

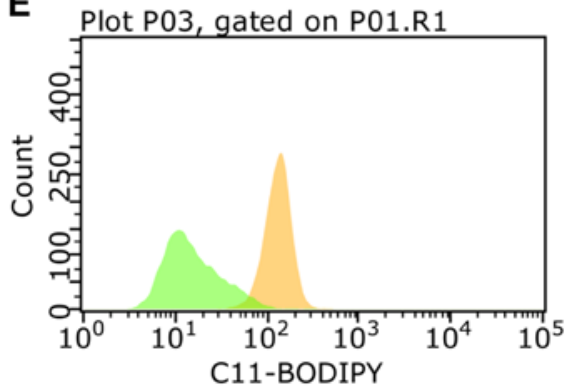

G

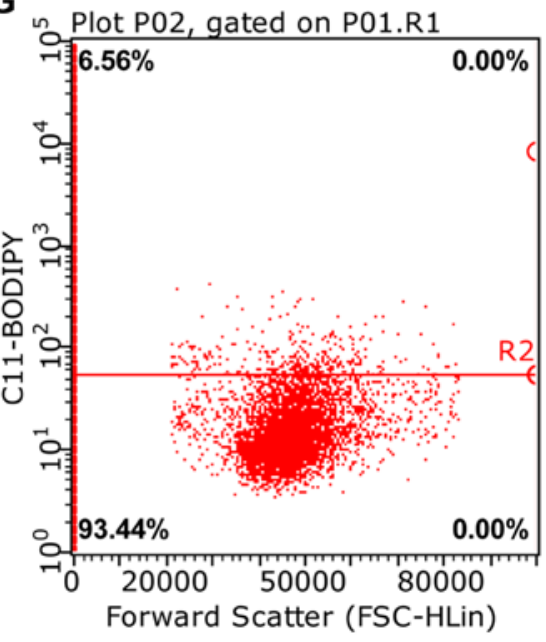

B

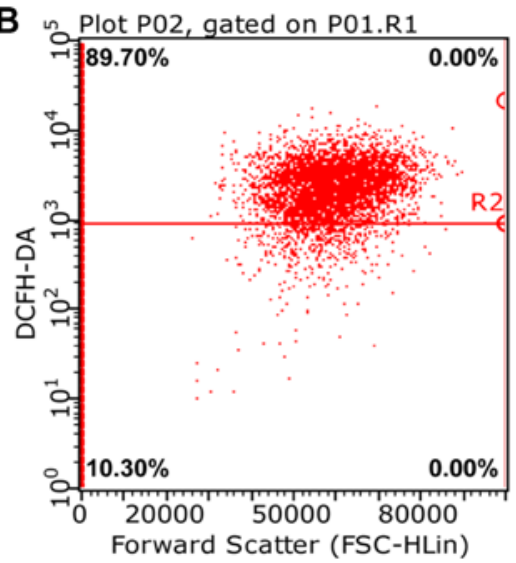

D

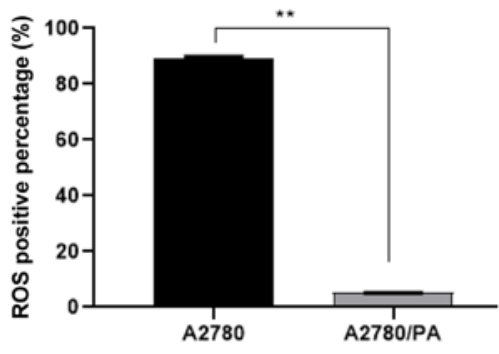

F n Plot P02, gated on P01.R1

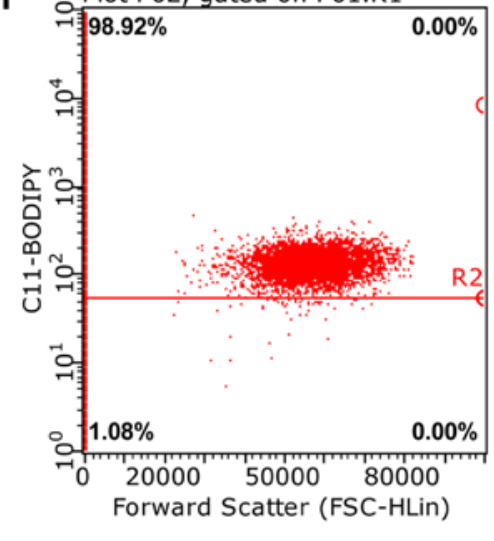

H

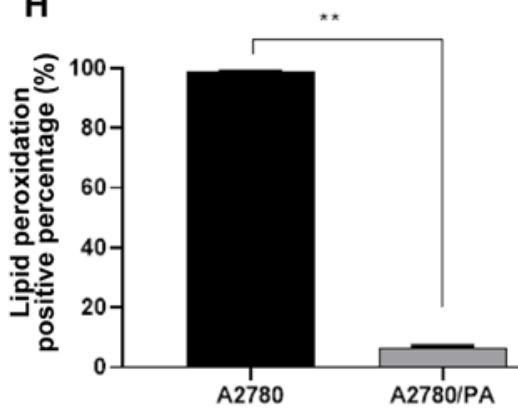

Figure 8. A2780/PA cells generate less reactive oxygen species and lipid peroxidation compared with A2780 cells, following treatment with paclitaxel. (A-D) ROS levels were analysed using DCFH-DA. (E-H) Lipid peroxidation level was analysed using C11-BODIPY. (A and E) Green area represents A2780/PA with paclitaxel treatment, whereas yellow area represents A2780 with paclitaxel treatment. (B, C, F and G) The dots above the R2 line mark the percentage of positive cells. ${ }^{* *} \mathrm{P}<0.01$ vs. A2780.

were used to analyse the GSE73935 chip dataset downloaded from the GEO database. The dataset included four different ovarian cancer chemotherapy-resistant cell lines and one chemotherapy-sensitive cell line. DEGs and overlapping genes were screened and PPI analysis of these genes yielded crucial genes that were then validated.

The current clinical guidelines recommend that the first-line chemotherapy drugs for advanced ovarian cancer should be 
cisplatin combined with paclitaxel, and that cisplatin plus doxorubicin can be used as a standard replacement for the former. If the patient has an allergic reaction to paclitaxel, topotecan is the preferred alternative $(3,4)$. Although the standard treatment for patients with ovarian cancer is cisplatin plus paclitaxel, other factors such as histology, distribution of disease, asymptomatic versus symptomatic, toxicity profile, genetic signature, vulnerable and elderly patients, and patient desire (for example to avoid hair loss) need to be taken into account (30).

The present study was based on an analysis of the genetic signature, which refers to the effect of genetic level on drug resistance, and it carries the largest weight among all these factors. Therefore, the results have high reference value for clinical decision-making and future research. By identifying DEGs through an integrated bioinformatics analysis of signalling pathways in ovarian cancer, as well as validating these findings using immunohistochemistry results and survival analysis curves, we hypothesise that data-driven clinical decisions are feasible.

Overlapping DEGs have the potential to indicate what genes are abnormally expressed in cells resistant to two or more chemotherapy drugs. The higher the number of overlapping DEGs between different drugs, the more likely the patient is to show resistance to one drug after showing resistance to another. Cisplatin combined with paclitaxel had the highest number of overlapping genes at 65 . Therefore, the results from the present study suggested that when patients subsequently develop cisplatin resistance, the likelihood of these patients to additionally acquire resistance to paclitaxel is high.

The exclusive overlapping DEGs ratio refers to the ratio of DEGs that are only resistant to two chemotherapeutic drugs among the four chemotherapeutic drugs The smaller the ratio, the more likely individual patients who are resistant to such combinations of drugs will be resistant to other combinations of drugs. Cisplatin combined with doxorubicin had the smallest proportion of exclusive consensus genes at only $16.2 \%$. Although some studies suggest that it can be used as a standard replacement $(4,31,32)$, when it is used as first-line therapy, with the emergence of drug resistance, the possibility of multi-drug resistance increases, to ensure more drug choices are available for subsequent treatment, this combination is not recommended for first-line chemotherapy.

After undergoing tumour resection, patients can be tested for protein and gene expression by immunohistochemistry and other methods. The present study identified eight genes that can guide individualised treatment according to their expression. EDIL3, an extracellular matrix protein associated with vascular morphogenesis and remodelling, is commonly upregulated in multiple types of human cancers and is correlated with apoptosis and tumour growth (33). Jiang et al (34) reported that EDIL3 exerts its antiapoptotic effect by altering the protein expression of the Bcl-2 family. The present study found that among patients with ovarian cancer with a high expression of EDIL3, those receiving a regimen of cisplatin plus paclitaxel had a poor prognosis, and thus this chemotherapy is not recommended.

$N R A S$ mutations are present in multiple cancers, and high expression of mutant NRAS in low-grade serous ovarian carcinomas leads to uncontrolled RAS/MAPK signalling (35). The current study found that patients with ovarian cancer who received cisplatin plus paclitaxel had a poorer prognosis when non-mutated NRAS was expressed at lower levels, thus this regimen is not recommended.

$P R O C R$ is known for promoting the migration of ovarian cancer cells (36). Previous studies have suggested that PROCR induces the activation of ERK and PI3K-Akt-mTOR signals to promote tumour growth $(37,38)$. The present study also found that the prognosis of patients with ovarian cancer, with high PROCR expression, and those receiving cisplatin combined with paclitaxel chemotherapy regimen was poor, and therefore this chemotherapy regimen should not be used, as it is unlikely to promote patient benefit.

HAPLN1 is known for activating NF- $\kappa \mathrm{B}$ activity to increase myeloma resistance (39), and is associated with the prognosis of several types of tumour $(40,41)$. To date there are no reports associating this gene with ovarian cancer. In the current study, the prognosis of ovarian cancer patients with high HAPLN1 expression who were receiving cisplatin combined with paclitaxel was found to be poor, but the prognosis in other chemotherapy groups was better. Combined with the microarray results, this gene was highly expressed in cisplatin-, doxorubicin- and topotecan-resistant cells, but was weakly expressed in paclitaxel-resistant cells. Therefore, for patients with high expression of HAPLN1, cisplatin combined with paclitaxel is not recommended. Instead, a combined chemotherapy regimen based on paclitaxel may be administered.

In cisplatin-resistant ovarian cancer cells, IGFIR is highly expressed (42), and the inhibition of IGFIR signalling pathway reverses cisplatin resistance in ovarian cancer $(43,44)$. These previous findings are consistent with the results of the present study, that suggest that the cisplatin-based chemotherapy regimen is also shown to not be beneficial in tumours expressing high levels of $I G F 1 R$.

The inhibition of $C D K N 2 A$ suppressed cell proliferation, invasion and migration abilities, while inducing apoptosis and sensitivity to paclitaxel in breast cancer (45). This supports the results of the present study that patients with high $C D K N 2 A$ expression levels were found to have a poor prognosis, therefore a paclitaxel-based regimen is not recommended in such patients.

CD53 promotes B cell receptor-dependent protein kinase $\mathrm{C}$ signalling (46), and increases tumour growth in $\mathrm{Cd} 53^{-1-}$ mice (47). Patients with low CD53 expression levels were found to have a poor prognosis. Therefore, cisplatin combined with doxorubicin is not recommended.

A pathway composed of the secreted SLIT glycoproteins and their ROBO receptors has the dual role of carcinogenesis and tumour suppression in human cancer (48). A previous study has shown that $\mathrm{ROBO} 2$ is downregulated in ovarian cancer as a tumour suppressor gene, and SLIT/ROBO activity can induce programmed cell death through the activation of caspase-3 in ovarian tumour cell lines (49). These findings support the results of the present study that patients with low $\mathrm{ROBO} 2$ expression were found to have a poor prognosis. Thus, these patients are not recommended to undergo cisplatin plus topotecan chemotherapy.

In addition, ROS levels and lipid peroxidation in A2780/PA cells were significantly lower than in A2780 after administration of paclitaxel, indicating that drug-resistant cell lines enhance their resistance to chemotherapeutic drugs by blocking the 
production of ROS and inducing lipid peroxidation. Previous studies have demonstrated that increased levels of ROS and lipid peroxidation can damage mitochondria and DNA, which in turn can affect cancer drug resistance $(25,26,29)$. Therefore, we hypothesised that the DEGs of paclitaxel-resistant cells proposed in this study may affect cell metabolism through mitochondrial functions and oxidative stress levels to promote resistance, which might have implications for future research directions.

In conclusion, using an integrated bioinformatics analysis for microarray datasets, eight crucial genes were identified, EDIL3, NRAS, HAPLN1, PROCR, CD53, CDKN2A, IGF1R and $R O B O 2$, that can indicate the most appropriate chemotherapy drugs for ovarian cancer in order to minimise the occurrence of ovarian cancer drug resistance enabling improved precision medicine. In addition, this study proposed recommendations for clinical drug use through an integrated bioinformatics analysis of microarray datasets. These findings could contribute to further basic research and clinical trial design. Data-driven clinical decisions have the potential to greatly promote the transparency and reproducibility of diagnoses and treatments. These findings could also provide new insights into genomic personalised medicine and survival prediction for ovarian cancer.

\section{Acknowledgements}

Not applicable.

\section{Funding}

This work was supported by The National Natural Science Foundation of China (grant no. 81772794), The National Natural Science Foundation of China (grant no. 81672948), The National Natural Science Foundation of China (grant no. 81472419), The Jilin Provincial Industrial Innovation Project (grant no. 2018C052-7), The Jilin Provincial Research Foundation for the Development of Science and Technology Projects (grant no. 20191004004TC), and The Fundamental Research Funds of Central Universities, Jilin University.

\section{Availability of data and materials}

The datasets used and/or analyzed during the present study are available from the corresponding author upon reasonable request.

\section{Authors' contributions}

DY drafted the initial manuscript and performed the experiments. HZ acquired and analyzed the data. HS revised the manuscript and assisted in some experiments. RT and MX assisted in the data processing, and reviewed and edited the manuscript. LS and YL supervised the research work, participated in designing the study and revised the manuscript. All authors read and approved the final manuscript.

\section{Ethics approval and consent to participate}

Not applicable.

\section{Patient consent for publication}

Not applicable.

\section{Competing interests}

The authors declare that they have no competing interests.

\section{References}

1. Siegel RL, Miller KD and Jemal A: Cancer statistics, 2020. CA Cancer J Clin 70: 7-30, 2020.

2. Terraneo N, Jacob F, Dubrovska A and Grünberg J: Novel therapeutic strategies for ovarian cancer stem cells. Front Oncol 10: 319,2020

3. Marth C, Reimer D and Zeimet AG: Front-line therapy of advanced epithelial ovarian cancer: Standard treatment. Ann Oncol 28 (suppl_8): viii36-viii39, 2017.

4. Pignata S, Lauraine EP, du Bois A and Pisano C: Pegylated liposomal doxorubicin combined with carboplatin: A rational treatment choice for advanced ovarian cancer. Crit Rev Oncol Hematol 73: 23-30, 2010.

5. Morgan RJ Jr, Armstrong DK, Alvarez RD, Bakkum-Gamez JN, Behbakht K, Chen LM, Copeland L, Crispens MA, DeRosa M, Dorigo O, et al: Ovarian cancer, version 1.2016, NCCN clinical practice guidelines in oncology. J Natl Compr Canc Netw 14: 1134-1163, 2016.

6. Rojas V, Hirshfield KM, Ganesan S and Rodriguez-Rodriguez L: Molecular characterization of epithelial ovarian Cancer: Implications for diagnosis and treatment. Int J Mol Sci 17: pii: E2113, 2016.

7. Thomas A and Pommier Y: Targeting Topoisomerase I in the Era of Precision Medicine. Clin Cancer Res 25: 6581-6589, 2019.

8. Dasari S and Tchounwou PB: Cisplatin in cancer therapy: Molecular mechanisms of action. Eur J Pharmacol 740: 364-378, 2014.

9. Wenningmann N, Knapp M, Ande A, Vaidya TR and Ait-Oudhia S: Insights into doxorubicin-induced cardiotoxicity: Molecular mechanisms, preventive strategies, and early monitoring. Mol Pharmacol 96: 219-232, 2019.

10. Zhu L and Chen L: Progress in research on paclitaxel and tumor immunotherapy. Cell Mol Biol Lett 24: 40, 2019.

11. Hoskins P, Eisenhauer E, Vergote I, Dubuc-Lissoir J, Fisher B, Grimshaw R, Oza A, Plante M, Stuart G and Vermorken J: Phase II feasibility study of sequential couplets of Cisplatin/Topotecan followed by paclitaxel/cisplatin as primary treatment for advanced epithelial ovarian cancer: A National Cancer Institute of Canada Clinical Trials Group Study. J Clin Oncol 18: 4038-4044, 2000.

12. Brotto L, Brundage M, Hoskins P, Vergote I, Cervantes A, Casado HA, Poveda A, Eisenhauer E and Tu D; Gynecologic Cancer Intergroup Study of NCIC Clinical Trials Group (NCIC CTG); European Organization for Research and Treatment of Cancer: Randomized study of sequential cisplatin-topotecan/carboplatin-paclitaxel versus carboplatin-paclitaxel: Effects on quality of life. Support Care Cancer 24: 1241-1249, 2016.

13. Hoskins P, Vergote I, Cervantes A, Tu D, Stuart G, Zola P, Poveda A, Provencher D, Katsaros D, Ojeda B, et al: Advanced ovarian cancer: Phase III randomized study of sequential cisplatin-topotecan and carboplatin-paclitaxel vs carboplatin-paclitaxel. J Natl Cancer Inst 102: 1547-1556, 2010.

14. Antman E, Weiss S and Loscalzo J: Systems pharmacology, pharmacogenetics, and clinical trial design in network medicine. Wiley Interdiscip Rev Syst Biol Med 4: 367-383, 2012.

15. Garralda E, Dienstmann R, Piris-Giménez A, Braña I, Rodon J and Tabernero J: New clinical trial designs in the era of precision medicine. Mol Oncol 13: 549-557, 2019.

16. Pignata S, Scambia G, Ferrandina G, Savarese A, Sorio R, Breda E, Gebbia V, Musso P, Frigerio L, Del Medico P, et al: Carboplatin plus paclitaxel versus carboplatin plus pegylated liposomal doxorubicin as first-line treatment for patients with ovarian cancer: the MITO-2 randomized phase III trial. J Clin Oncol 29: 3628-3635, 2011.

17. Kurtz JE, Kaminsky MC, Floquet A, Veillard AS, Kimmig R, Dorum A, Elit L, Buck M, Petru E, Reed N, et al: Ovarian cancer in elderly patients: Carboplatin and pegylated liposomal doxorubicin versus carboplatin and paclitaxel in late relapse: A Gynecologic Cancer Intergroup (GCIG) CALYPSO sub-study. Ann Oncol 22: 2417-2423, 2011. 
18. Sehouli J, Chekerov R, Reinthaller A, Richter R, Gonzalez-Martin A, Harter P, Woopen H, Petru E, Hanker LC, Keil E, et al: Topotecan plus carboplatin versus standard therapy with paclitaxel plus carboplatin (PC) or gemcitabine plus carboplatin (GC) or pegylated liposomal doxorubicin plus carboplatin (PLDC): A randomized phase III trial of the NOGGO-AGO-Study Group-AGO Austria and GEICO-ENGOT-GCIG intergroup study (HECTOR). Ann Oncol 27: 2236-2241, 2016.

19. Bolis G, Scarfone G, Raspagliesi F, Mangili G, Danese S, Scollo P, Lo Russo D, Villa A, Aimone PD and Scambia G: Paclitaxel/carboplatin versus topotecan/paclitaxel/carboplatin in patients with FIGO suboptimally resected stage III-IV epithelial ovarian cancer a multicenter, randomized study. Eur J Cancer 46 2905-2912, 2010.

20. Galluzzi L, Vitale I, Michels J, Brenner C, Szabadkai G Harel-Bellan A, Castedo M and Kroemer G: Systems biology of cisplatin resistance: Past, present and future. Cell Death Dis 5: e1257, 2014.

21. Livak KJ and Schmittgen TD: Analysis of relative gene expression data using real-time quantitative PCR and the 2(-Delta Delta C(T)) method. Methods 25: 402-408, 2001.

22. Schmittgen TD and Livak KJ: Analyzing real-time PCR data by the comparative C(T) method. Nat Protoc 3: 1101-1108, 2008.

23. Irizarry RA, Hobbs B, Collin F, Beazer-Barclay YD, Antonellis KJ, Scherf U and Speed TP: Exploration, normalization, and summaries of high density oligonucleotide array probe level data. Biostatistics 4: 249-264, 2003.

24. Armstrong DK, Alvarez RD, Bakkum-Gamez JN, Barroilhet L, Behbakht K, Berchuck A, Berek JS, Chen LM, Cristea M, DeRosa M, et al: NCCN Guidelines Insights: Ovarian Cancer, Version 1.2019. J Natl Compr Canc Netw 17: 896-909, 2019.

25. Zheng $X$ and Li H: TKTL1 modulates the response of paclitaxel-resistant human ovarian cancer cells to paclitaxel. Biochem Biophys Res Commun 503: 572-579, 2018.

26. Okon IS and Zou MH: Mitochondrial ROS and cancer drug resistance: Implications for therapy. Pharmacol Res 100: 170-174, 2015.

27. Chen G, Wang F, Trachootham D and Huang P: Preferential killing of cancer cells with mitochondrial dysfunction by natural compounds. Mitochondrion 10: 614-625, 2010.

28. Okon IS, Coughlan KA, Zhang M, Wang Q and Zou MH: Gefitinib-mediated reactive oxygen specie (ROS) instigates mitochondrial dysfunction and drug resistance in lung cancer cells. J Biol Chem 290: 9101-9110, 2015.

29. Środa-Pomianek K, Michalak K, Światek P, Poła A, Palko-Łabuz A and Wesołowska O: Increased lipid peroxidation, apoptosis and selective cytotoxicity in colon cancer cell line LoVo and its doxorubicin-resistant subline $\mathrm{LoVo} / \mathrm{Dx}$ in the presence of newly synthesized phenothiazine derivatives. Biomed Pharmacother 106: 624-636, 2018.

30. Harter P, Hilpert F, Mahner S, Heitz F, Pfisterer J and du Bois A Systemic therapy in recurrent ovarian cancer: Current treatment options and new drugs. Expert Rev Anticancer Ther 10: 81-88, 2010.

31. Tempfer CB, Hartmann F, Hilal $\mathrm{Z}$ and Rezniczek GA: Intraperitoneal cisplatin and doxorubicin as maintenance chemotherapy for unresectable ovarian cancer: A case report. BMC Cancer 17: 26, 2017.

32. Tempfer CB, Giger-Pabst U, Seebacher V, Petersen M, Dogan A and Rezniczek GA: A phase I, single-arm, open-label, dose escalation study of intraperitoneal cisplatin and doxorubicin in patients with recurrent ovarian cancer and peritoneal carcinomatosis. Gynecol Oncol 150: 23-30, 2018.

33. Feng MX, Ma MZ, Fu Y, Li J, Wang T, Xue F, Zhang JJ, Qin WX, Gu JR, Zhang ZG and Xia Q: Elevated autocrine EDIL3 protects hepatocellular carcinoma from anoikis through RGD-mediated integrin activation. Mol Cancer 13: 226, 2014.

34. Jiang SH, Wang Y, Yang JY, Li J, Feng MX, Wang YH, Yang XM, He P, Tian GA, Zhang XX, et al: Overexpressed EDIL3 predicts poor prognosis and promotes anchorage-independent tumor growth in human pancreatic cancer. Oncotarget 7: 4226-4240, 2016.
35. Etemadmoghadam D, Azar WJ, Lei Y, Moujaber T, Garsed DW, Kennedy CJ, Fereday S, Mitchell C, Chiew YE, Hendley J, et al: EIFIAX and NRAS mutations co-occur and cooperate in low-grade serous ovarian carcinomas. Cancer Res 77: 4268-4278, 2017.

36. Althawadi H, Alfarsi H, Besbes S, Mirshahi S, Ducros E, Rafii A, Pocard M, Therwath A, Soria J and Mirshahi M: Activated protein $\mathrm{C}$ upregulates ovarian cancer cell migration and promotes unclottability of the cancer cell microenvironment. Oncol Rep 34: 603-609, 2015.

37. Polyak K and Metzger Filho O: SnapShot: Breast cancer. Cancer Cell 22: 562-562.e1, 2012.

38. Wang D, Liu C, Wang J, Jia Y, Hu X, Jiang H, Shao ZM and Zeng YA: Protein $C$ receptor stimulates multiple signaling pathways in breast cancer cells. J Biol Chem 293: 1413-1424, 2018.

39. Huynh M, Pak C, Markovina S, Callander NS, Chng KS, Wuerzberger-Davis SM, Bakshi DD, Kink JA, Hematti P, Hope C, et al: Hyaluronan and proteoglycan link protein 1 (HAPLN1) activates bortezomib-resistant NF- $\kappa \mathrm{B}$ activity and increases drug resistance in multiple myeloma. J Biol Chem 293: 2452-2465, 2018.

40. Ecker BL, Kaur A, Douglass SM, Webster MR, Almeida FV, Marino GE, Sinnamon AJ, Neuwirth MG, Alicea GM, Ndoye A, et al: Age-related Changes in HAPLN1 increase lymphatic permeability and affect routes of melanoma metastasis. Cancer Discov 9: 82-95, 2019.

41. Yau C, Esserman L, Moore DH, Waldman F, Sninsky J and Benz CC: A multigene predictor of metastatic outcome in early stage hormone receptor-negative and triple-negative breast cancer. Breast Cancer Res 12: R85, 2010.

42. Eckstein N, Servan K, Hildebrandt B, Pölitz A, von Jonquières G, Wolf-Kümmeth S, Napierski I, Hamacher A, Kassack MU, Budczies J, et al: Hyperactivation of the insulin-like growth factor receptor I signaling pathway is an essential event for cisplatin resistance of ovarian cancer cells. Cancer Res 69: 2996-3003, 2009.

43. Du J, Shi HR, Ren F, Wang JL, Wu QH, Li X and Zhang RT: Inhibition of the IGF signaling pathway reverses cisplatin resistance in ovarian cancer cells. BMC Cancer 17: 851, 2017.

44. Zhang Y, Huang S, Guo Y and Li L: MiR-1294 confers cisplatin resistance in ovarian Cancer cells by targeting IGF1R. Biomed Pharmacother 106: 1357-1363, 2018.

45. Wang L, Zhan X, Shen X, Li M, Yang J, Yu W, Chen H, Jin B and Mao Z: P16 promotes the growth and mobility potential of breast cancer both in vitro and in vivo: the key role of the activation of IL-6/JAK2/STAT3 signaling. Mol Cell Biochem 446: 137-148, 2018.

46. Zuidscherwoude M, Dunlock VE, van den Bogaart G, van Deventer SJ, van der Schaaf A, van Oostrum J, Goedhart J, In 't Hout J, Hämmerling GJ, Tanaka S, et al: Tetraspanin microdomains control localized protein kinase $\mathrm{C}$ signaling in $\mathrm{B}$ cells. Sci Signal 10: pii: eaag2755, 2017.

47. Schaper F and van Spriel AB: Antitumor immunity is controlled by tetraspanin proteins. Front Immunol 9: 1185, 2018

48. Jiang Z, Liang G, Xiao Y, Qin T, Chen X, Wu E, Ma Q and Wang Z: Targeting the SLIT/ROBO pathway in tumor progression: molecular mechanisms and therapeutic perspectives. Ther Adv Med Oncol 11: 1758835919855238, 2019.

49. Dickinson RE and Duncan WC: The SLIT-ROBO pathway: A regulator of cell function with implications for the reproductive system. Reproduction 139: 697-704, 2010.

This work is licensed under a Creative Commons Attribution-NonCommercial-NoDerivatives 4.0 International (CC BY-NC-ND 4.0) License. 